\title{
Multimodal MRI Neuroimaging Biomarkers for Cognitive Normal Adults, Amnestic Mild Cognitive Impairment, and Alzheimer's Disease
}

\author{
Ai-Ling Lin, ${ }^{1}$ Angela R. Laird, ${ }^{1}$ Peter T. Fox, ${ }^{1}$ and Jia-Hong Gao ${ }^{2}$ \\ ${ }^{1}$ Research Imaging Institute, The University of Texas Health Science Center at San Antonio, \\ 7703 Floyd Curl Drive, San Antonio, TX 78229, USA \\ ${ }^{2}$ Departments of Radiology, Psychiatry, and Behavioral Neuroscience and Brain Research Imaging Center, The University of Chicago, \\ 5841 South Maryland, MC 2026, Chicago, IL 60637, USA \\ Correspondence should be addressed to Ai-Ling Lin, lina3@uthscsa.edu and Jia-Hong Gao, jgao@uchicago.edu
}

Received 16 March 2011; Revised 11 May 2011; Accepted 8 June 2011

Academic Editor: Andrea Cherubini

Copyright (c) 2012 Ai-Ling Lin et al. This is an open access article distributed under the Creative Commons Attribution License, which permits unrestricted use, distribution, and reproduction in any medium, provided the original work is properly cited.

Multimodal magnetic resonance imaging (MRI) techniques have been developed to noninvasively measure structural, metabolic, hemodynamic and functional changes of the brain. These advantages have made MRI an important tool to investigate neurodegenerative disorders, including diagnosis, disease progression monitoring, and treatment efficacy evaluation. This paper discusses recent findings of the multimodal MRI in the context of surrogate biomarkers for identifying the risk for AD in normal cognitive (NC) adults, brain anatomical and functional alterations in amnestic mild cognitive impairment (aMCI), and Alzheimer's disease (AD) patients. Further developments of these techniques and the establishment of promising neuroimaging biomarkers will enhance our ability to diagnose $\mathrm{MMCI}$ and $\mathrm{AD}$ in their early stages and improve the assessment of therapeutic efficacy in these diseases in future clinical trials.

\section{Introduction}

Aging is the greatest risk factor for neurodegenerative disorders in general, but specifically for Alzheimer's disease (AD). With the increasing life expectancy in developed countries, the incidence of $\mathrm{AD}$, and consequently its socioeconomic impact, is growing. AD currently affects about 4.5 million Americans, which costs the USA economy more than $\$ 100$ billion each year. The number of $\mathrm{AD}$ patients is projected to increase to $11-16$ millions by 2050 , with a cost exceeding $\$ 380$ billion per year $[1,2]$.

To identify $\mathrm{AD}$ and monitor disease progression, neuropsychological tests such as the Mini-Mental State Exam (MMSE) and the cognitive subscale of the Alzheimers disease assessment (ADAS Cog) [3] are currently the most commonly used strategies. However, these tests have several limitations, as follows. MMSE is criticized by its marginal or absent assessment of some cognitive abilities that are affected early in the course of Alzheimer's disease or other dementing disorders (e.g., limited memory and verbal fluency items and no problem solving or judgment items), and its relative insensitivity to very mild cognitive decline, particularly in highly educated individuals. ADAS Cog is limited by its relatively poor test-retest reliability, which likely reflects the influence of other factors on the patients' performance (e.g., the patients' mood). Furthermore, these tests are not able to distinguish the risk for $\mathrm{AD}$ in preclinical groups (cognitively normal elderly adults) or predict the conversion to $\mathrm{AD}$ from preclinical and mild cognitive impairment (MCI) groups.

The National Institute of Aging (NIA) has recently announced the revised clinical diagnostic criteria for $\mathrm{AD}$ dementia for the first time in 27 years (http://www.nih .gov/news/health/apr2011/nia-19.htm). Instead of addressing the disease and describing only later stages when symptoms of dementia are already evident, the updated guidelines cover the full spectrum of the disease as it gradually changes over many years. They describe (i) the earliest preclinical stages of the disease, (ii) MCI, and 
(iii) dementia due to Alzheimer's pathology. Importantly, the guidelines now address the use of imaging and biomarkers in blood and spinal fluid that may help determine whether changes in the brain and those in body fluids are due to AD.

In this paper, we will focus on the imaging biomarkers as addressed in the new criteria. Specifically, we will discuss the surrogate biomarkers developed by the multimodal magnetic resonance imaging $(\mathrm{MRI})$ methods for identifying the risk for $\mathrm{AD}$ in normal cognitive (NC) adults; brain anatomical and functional alterations in amnestic MCI (aMCI) and AD patients.

\section{Brief Overview of Multimodal MRI Neuroimaging Biomarkers}

2.1. Structural Biomarkers. The high spatial resolution, sensitivity, and specificity of MRI (e.g., resolution: $0.8 \mathrm{~mm}$ isotropic; sensitivity: 80-94\%; specificity: 60-100\%) have made it a powerful tool to identify structural alterations and brain atrophy using volumetric measurements of the entire brain $[4,5]$. With advanced computer software, the neocortex of the brain on the MRI scans can be automatically subdivided into 32 gyral-based region of interests (ROIs) per hemisphere, including gray matter (GM), white matter (WM), and hippocampus volumes [6-8].

GM loss can also be determined by measuring cortical gray matter thickness (GMT). GMT is determined by calculating the three-dimensional distance from the outer cortical surface to the inner cortical GM-WM boundary using cortical modeling from the high-resolution MRI structural images (Figure 1). WM integrity can be assessed with diffusion tensor imaging (DTI). In brain tissues, the microscopic motion of water molecules is hindered by boundaries of tissue structure. In highly structured tissue such as WM, this motion is highly anisotropic and DTI provides directional information about it. Loss in WM structure results in the loss in anisotropy, which can be easily detected by DTI $[9,10]$.

2.2. Functional Biomarkers. Functional-based MRI can detect alterations and monitor disease progression related to brain metabolism, hemodynamics, and connectivity. Functional connectivity MRI ( $\mathrm{fcMRI}$ ) has been developed as a technique to determine the resting state brain connectivity as measured by the basal blood oxygenation-level-dependent (BOLD) signal. In its simplest form, functionally connected networks can be identified using a seed-based correlational approach, in which the average resting state time series from a region of interest is correlated with all other voxels in the brain $[2,11-14]$. In contrast to this correlational method, independent component analysis (ICA) is a more advanced multivariate analysis method that allows resting state fMRI data to be decomposed into sets of independent, intrinsic brain networks [15-17]. In either approach, each functional network's neuronal activity is associated with a hemodynamic response, which consists of an increase in cerebral blood flow $(\mathrm{CBF})$ and oxyhemoglobin and a rel- ative decrease in deoxyhemoglobin. The changes in the oxyhemoglobin-deoxyhemoglobin ratio result in changes in BOLD signal.

Neuronal activity is tightly coupled with CBF (as mentioned above); therefore, another approach to assess the disease progression of $\mathrm{AD}$ is to measure $\mathrm{CBF}$ (in the units of $\mathrm{mL} / 100 \mathrm{~g} / \mathrm{min})$. MR-based CBF measurements have been developed to investigate hemodynamic alteration in $\mathrm{AD}$, including arterial spin labeling (ASL) [18] and dynamic contrast techniques [19]. Compared with the traditional CBF measurements using single-photon emission computed tomography (SPECT) with Tc-99m radioactive tracers [20, 21 ], the absence of ionizing radiation or injection and the ability to obtain high quality anatomical images within the same scanning session make MRI-based CBF techniques attractive methods for the study of $\mathrm{AD}$, especially when repeated scans are needed for monitoring disease progression or assessing treatment effect.

Changes in neuronal activity during the progression of $\mathrm{AD}$ may be associated with the changes in brain metabolism. Brain metabolism can be measured with MR spectroscopy (MRS). Using proton $\left({ }^{1} \mathrm{H}\right)$ MRS, numerous metabolites related to brain functions can be determined, including $\mathrm{N}$ acetyl aspartate (NAA), myoinositol (MI), creatine, choline, glutamate, glutamine and lactate. NAA is present only within neural cell body, axons and dendrites, it is thus considered to be a marker of neuronal viability and function [22]. MI, on the other hand, has considerably higher concentration in glial cells and thus is often taken as a glial marker [23].

\section{Preclinical-Cognitively Normal Adults}

Beyond age, family history is the most significant risk factor for $\mathrm{AD}$, with maternal transmission being significantly more frequent than paternal transmission [24]. Biomarkers for $\mathrm{AD}$-associated pathological changes, including metabolic deficits and amyloid beta $(\mathrm{A} \beta)$ load, have been observed in cognitively normal individuals who have maternal history of late-onset $\mathrm{AD}$ (FHm) [25, 26].

Structural MRI has been used to assess brain volume changes for cognitively intact elderly individuals with $\mathrm{FHm}$, a paternal history of $\mathrm{AD}(\mathrm{FHp})$ and no parental history of $\mathrm{AD}$ (FH-) [27-29]. Compared with FH individuals, cognitively healthy subjects with a family history of late-onset $\mathrm{AD}$ had significantly decreased gray matter volume (GMV) in the precuneus, middle frontal, and superior frontal gyri (Figure 2; [27]). FHm subjects had even significantly smaller inferior frontal, middle frontal, precuneus, and lingual gyri compared with FH and FHp individuals (Figures 2 and 3) $[27,28]$.

Another chief known genetic factor for $\mathrm{AD}$ is $\varepsilon 4$ allele apolipoprotein $\mathrm{E}$ gene (APOE $\varepsilon 4$ ). Increasing age and carrying APOE $\varepsilon 4$ are well-established risk factors for $\mathrm{AD}$. Healthy older APOE $\varepsilon 4$ carriers, particularly $\varepsilon 4$ homozygotes, have demonstrated brain structure changes related to noncarriers. In a recent study with a longitudinal cohort of 1186 healthy elderly persons (65-89 years), Crivello et al. found that an annual rate of gray matter volume loss 

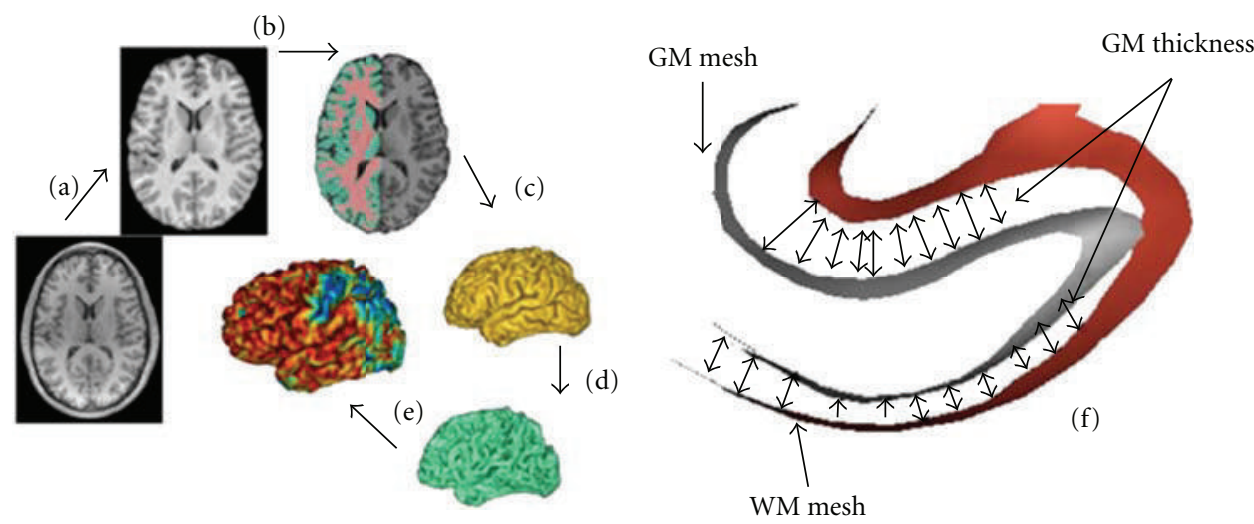

FIGURE 1: $\mathrm{T}_{1}$-weighted image processing pipeline consists of (a) skull stripping; (b) spatial normalization, RF homogeneity correction, and tissue segmentation; (c, d) extraction of GM and WM pial surfaces; (e, f) calculation of GMT.

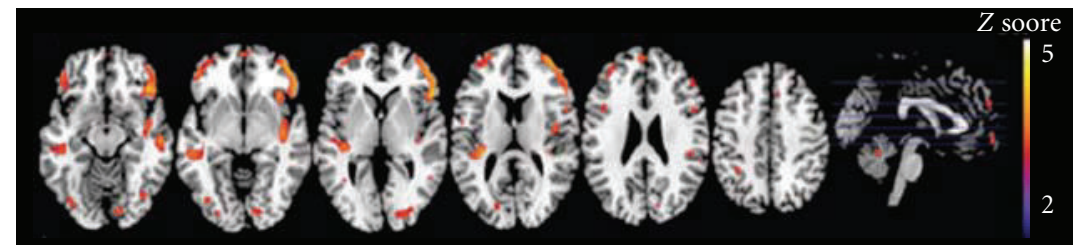

(a)

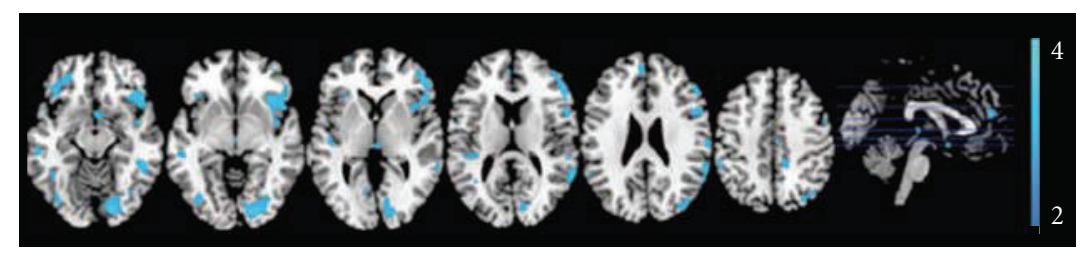

(b)

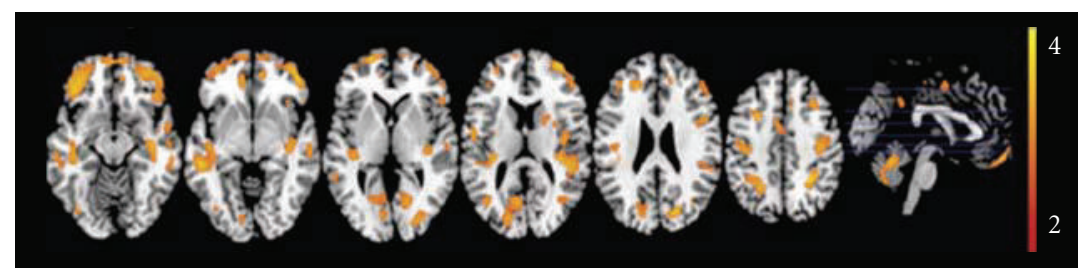

(c)

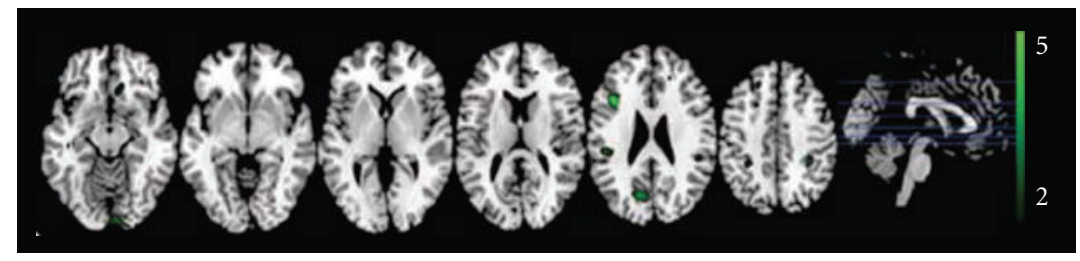

(d)

FIGURE 2: The first 2 rows display maps from subjects with a maternal family history of Alzheimer's disease (FHm) as compared with subjects with no family history (FH-) (a) and subjects with a paternal family history (FHp) (b). Row (c) shows gray matter volume (GMV) reductions in APOE $\varepsilon$ 4-negative FHm subjects compared with APOE $\varepsilon 4$-negative FH subjects. Statistical parametric maps showing GMV reductions in normal FHp subjects as compared with FH subjects are in row (d). Areas of GMV decrease are represented on purple-to-yellow, blue-tolight blue, dark orange-to-yellow, and green-to-light green color-coded scales for the 4 contrasts, reflecting $Z$ scores between 2 and 5 for the upper contrast and between 2 and 4 for the lower 3 contrasts. Areas of gray matter volume decrease are displayed on a standardized spatially normalized MRI (adapted with permission from [27]). 


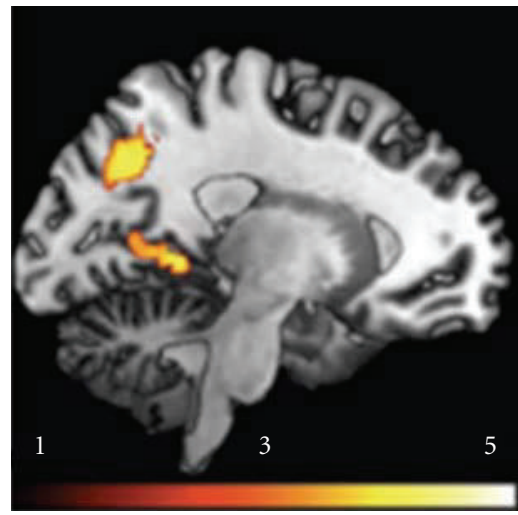

(a)

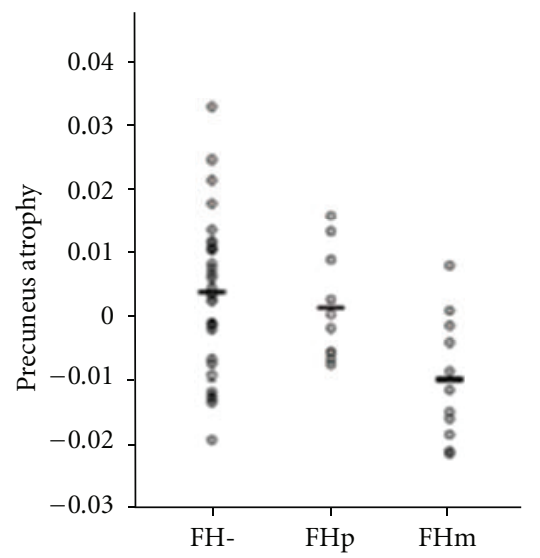

(b)

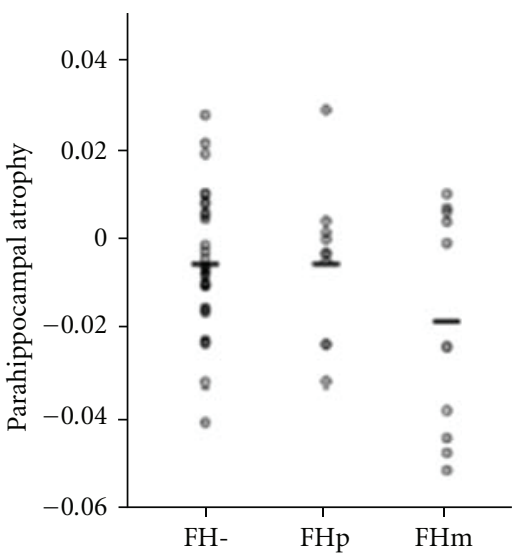

(c)

FIGURE 3: Increased regional atrophy in maternal history of Alzheimer's disease (FHm) group compared to subjects without family history of late-onset Alzheimer's disease (FH-) and paternal history of Alzheimer's disease (FHp) groups (adapted with permission from [28]).

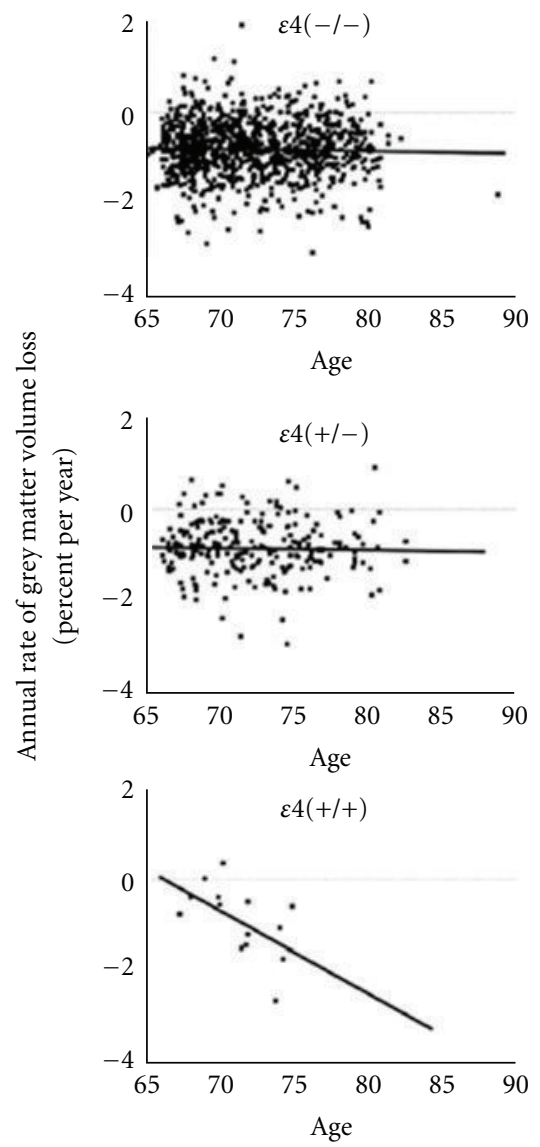

(a)

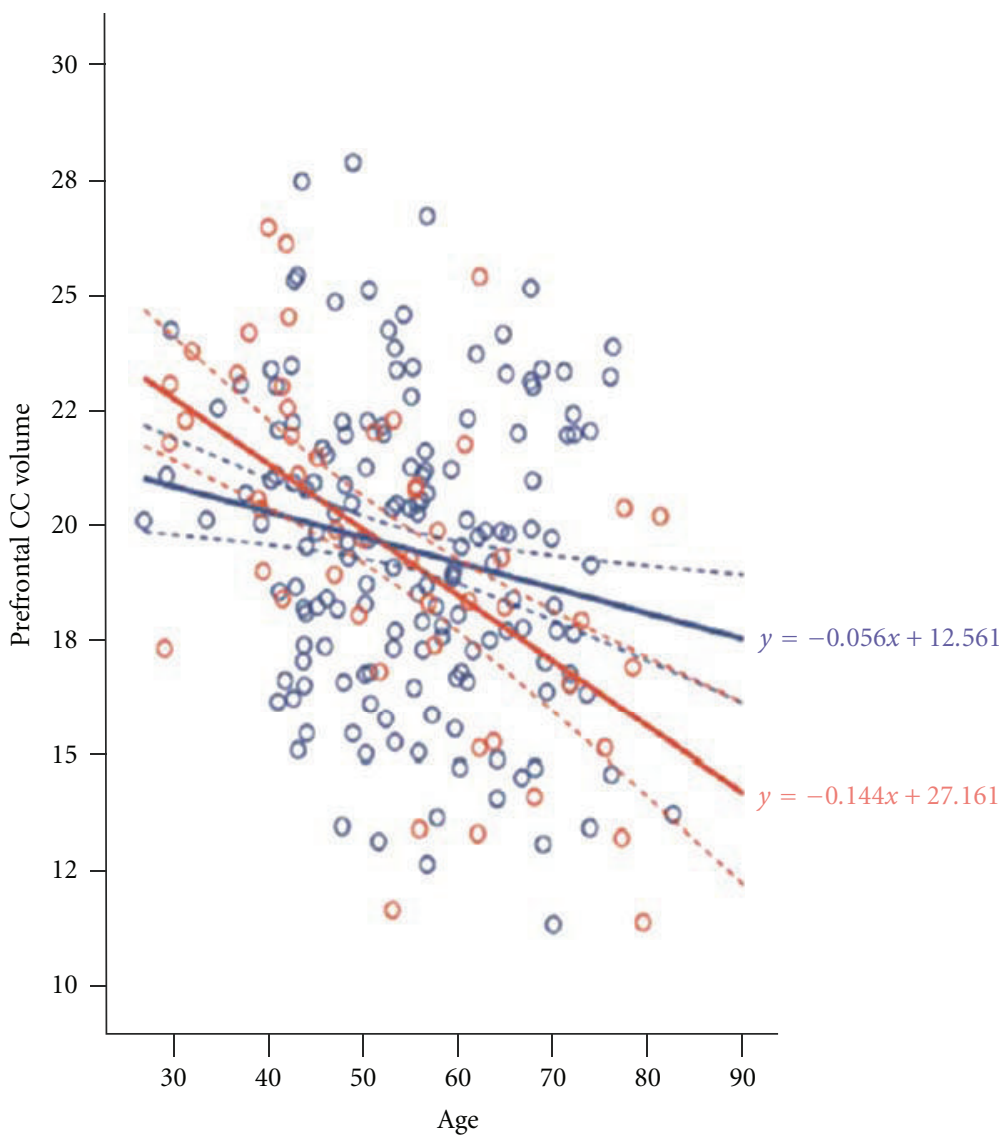

(b)

FIgURE 4: (a) Age effect on the longitudinal followup of GMV for the three APOE $\varepsilon 4$ groups, illustrating the significant interaction between age and the APOE $\varepsilon 4 . \varepsilon 4(-/-)$ : noncarriers for the APOE $\varepsilon 4$ allele, and $\varepsilon 4$ (+/-): heterozygous for the APOE $\varepsilon 4$ allele, $\varepsilon 4$ (+/+): homozygous for the APOE $\varepsilon 4$ allele (adapted with permission from [30]). (b) Regression of the normalized volume of the prefrontal callosal subregion on age in APOE $\varepsilon 4$ carriers (red) and noncarriers (blue). Thick lines show linear regression lines and dotted lines $95 \%$ mean confidence intervals of the slope. Formulas denote regression lines. Normalization was done to total intracranial volume (adapted with permission from [31]). 


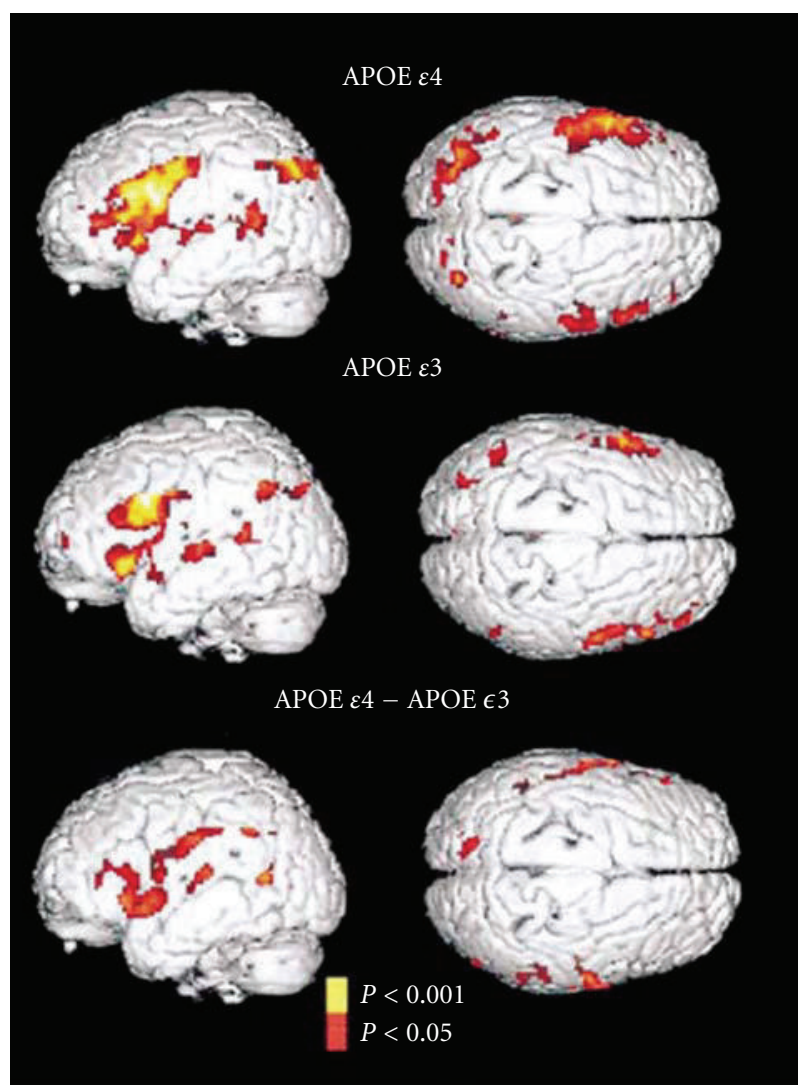

Figure 5: Statistical parametric maps of the brain used to assess subjects' performance on memory-activation tests in carriers of the APOE $\varepsilon 4$ allele and carriers of the APOE $\varepsilon 3$ allele. The signal intensity increased significantly in the left inferior frontal region, the right prefrontal cortex, the transverse temporal gyri bilaterally, and the left posterior temporal and inferior parietal regions in both groups. However, both the extent and the intensity of activation were greater among the carriers of the APOE $\varepsilon 4$ allele. The carriers of the APOE $\varepsilon 4$ allele also had significant increases in the left parahippocampal, the left dorsal prefrontal cortex, and in the inferior and superior parietal lobes and the anterior cingulate gyrus. Direct comparisons of the carriers of the APOE $\varepsilon 4$ allele and the carriers of the APOE $\varepsilon 3$ allele (bottom panel, which shows the difference between the carriers) further demonstrated the greater extent and magnitude of activity in the left prefrontal region and bilateral orbitofrontal, superior temporal, and inferior and superior parietal regions in the carriers of the APOE $\varepsilon 4$ allele (adapted with permission from [32]).

was seen in $\varepsilon 4$ homozygotes, whereas no age effect was seen in $\varepsilon 4$ heterozygotes and in noncarriers (Figure 4(a)) [30]. Similarly, $\varepsilon 4$ homozygotes had a significant larger rate of hippocampal volume loss than heterozygotes or noncarriers. In another anatomical study, Filippini et al. observed white matter atrophy, including corpus callosum (CC) volume and all subregions, in both APOE $\varepsilon 4$ carriers and noncarriers. However, the slope has been steeper in the APOE $\varepsilon 4$ carriers compared with the noncarriers particularly in the prefrontal region $(P=0.02)$ (Figure $4(\mathrm{~b}))$ [31].

In addition to structural changes, APOE $\varepsilon 4$ has also shown great impact on brain function. Memory is the first cognitive domain to be affected by $\mathrm{AD}$ [42], and impairments have been found in APOE $\varepsilon 4$ carriers relative to noncarriers $[43,44]$. Using functional MRI (fMRI), Bookheimer et al. observed that during a memory task in a group of healthy subjects (aged 47-82), APOE $\varepsilon 4$ carriers demonstrated significant increases in the left parahippocampal region, the left dorsal prefrontal cortex, the inferior-superior parietal lobes, and the anterior cingulate gyrus (Figure 5; [32]). In addition, the extent and the intensity of activation for the APOE $\varepsilon 4$ carriers were greater in the left inferior frontal region, the right prefrontal cortex, the transverse temporal gyri bilaterally, the left posterior temporal, and inferior parietal regions relative to the noncarriers (carriers of the APOE $\varepsilon 3$ allele). Direct comparisons of APOE $\varepsilon 4$ carriers and noncarriers further demonstrated the greater extent and magnitude of activity in the left prefrontal, bilateral orbitofrontal, and superior temporal regions. In carriers of the APOE $\varepsilon 4$ allele, it has been demonstrated in inferior and superior parietal regions.

In a younger group (mean age $=21-30$ ), APOE $\varepsilon 4$ carriers demonstrated increased task-induced brain activation in hippocampus relative to the noncarriers $[45,46]$. Overactivity of brain function has also been found in young APOE $\varepsilon 4$ carriers but disproportionately reduced with advancing age even before the onset of measurable memory impairment (Figure 6; [33]). In both age groups, a significant interaction has been found between age and APOE $\varepsilon 4$ status in the hippocampi, frontal pole, subcortical nuclei, middle temporal gyri, and cerebellum. These results have suggested that APOE genotype determines age-related changes in brain function, and greater activation reflects greater cognitive "effort" by APOE $\varepsilon 4$ carriers to obtain the same level of performance as the noncarriers, and/or reflect neuronal mechanism to compensate for processes, such as reduced synaptic plasticity, neuronal growth, or altered long-term potentiation in the carriers.

APOE $\varepsilon 4$ carriers have also shown disrupted resting state brain activity in the absence of $\mathrm{A} \beta$ or decreased CSF in cognitively normal elderly (mean age $=62$ ) using functional connectivity MRI method [11-13,47]. Similarly, young APOE $\varepsilon 4$ carriers (mean age $=21-30$ ), although had no difference in cognition and GM volume compared to their age-mated controls, showed increase in default mode network (involving medial temporal, medial prefrontal, and retrosplenial cortical areas) coactivation [46], suggesting that the function of these areas subject to the disease process in $\mathrm{AD}$ is modulated by APOE $\varepsilon 4$ allele at very early stage.

Taken together, these results provide evidence that influence of the genetic effect (familial and APOE $\varepsilon 4$ allele) on neurophysiological characteristics and the risk for AD can be detected using MRI decades prior to any clinical or neuropathological expression of neurodegenerative process.

\section{Mild Cognitive Impairment}

MCI is a transitional state between normal aging and dementia. MCI is a diagnosis given to individuals who experience memory problems greater than normally expected 


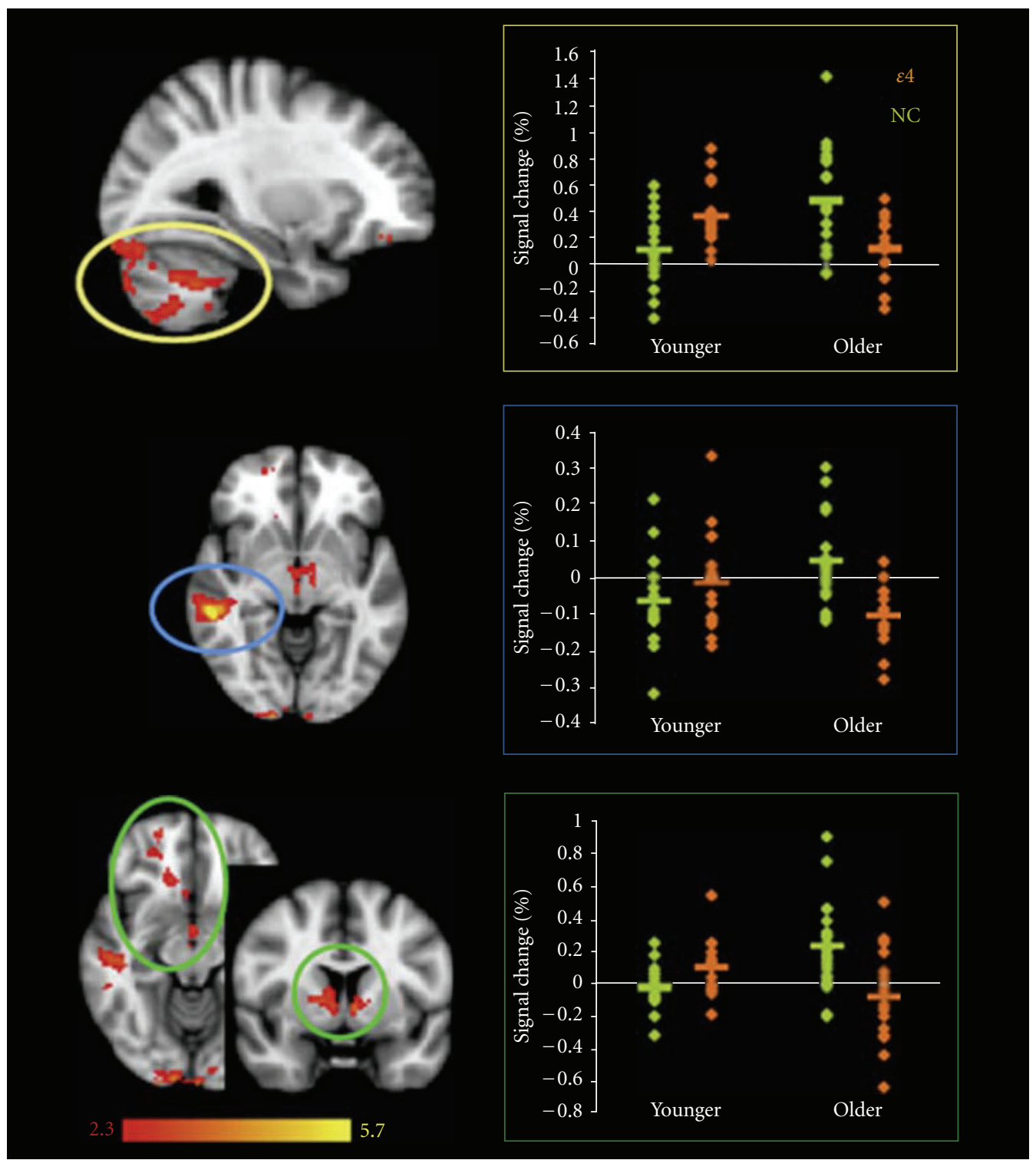

(a)

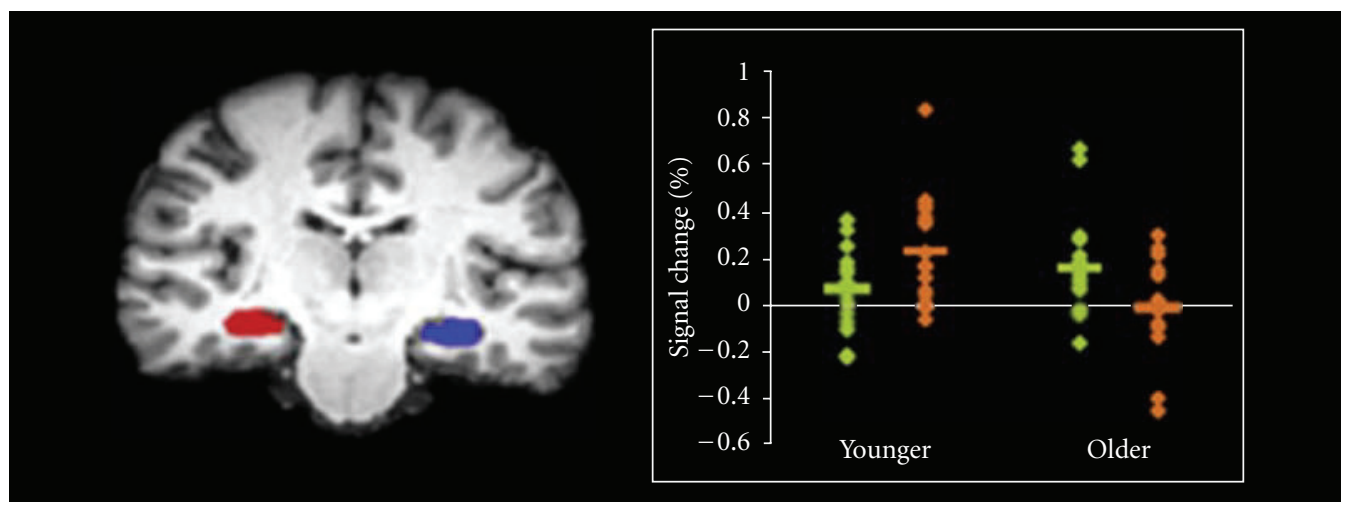

(b)

FIGURE 6: AGE by GENE interactions in the "novel versus familiar" contrast of the encoding task. (a) Regions showing significant interaction between AGE and GENE factors $(P<0.05$, corrected for multiple comparisons) with plots of percentage signal change in brain regions showing group-related differences where $\varepsilon 4$ (orange) defines $\varepsilon 4$-carriers and $\mathrm{NC}$ (green) defines noncarriers. (b) ROIs for the left and right hippocampi overlaid on a structural image (left) with associated plot of average hippocampal percentage signal change showing significant age-by-gene interaction (left hippocampus: $P=0.002$, right hippocampus: $P=0.003$ ) (adapted with permission from [33]). 


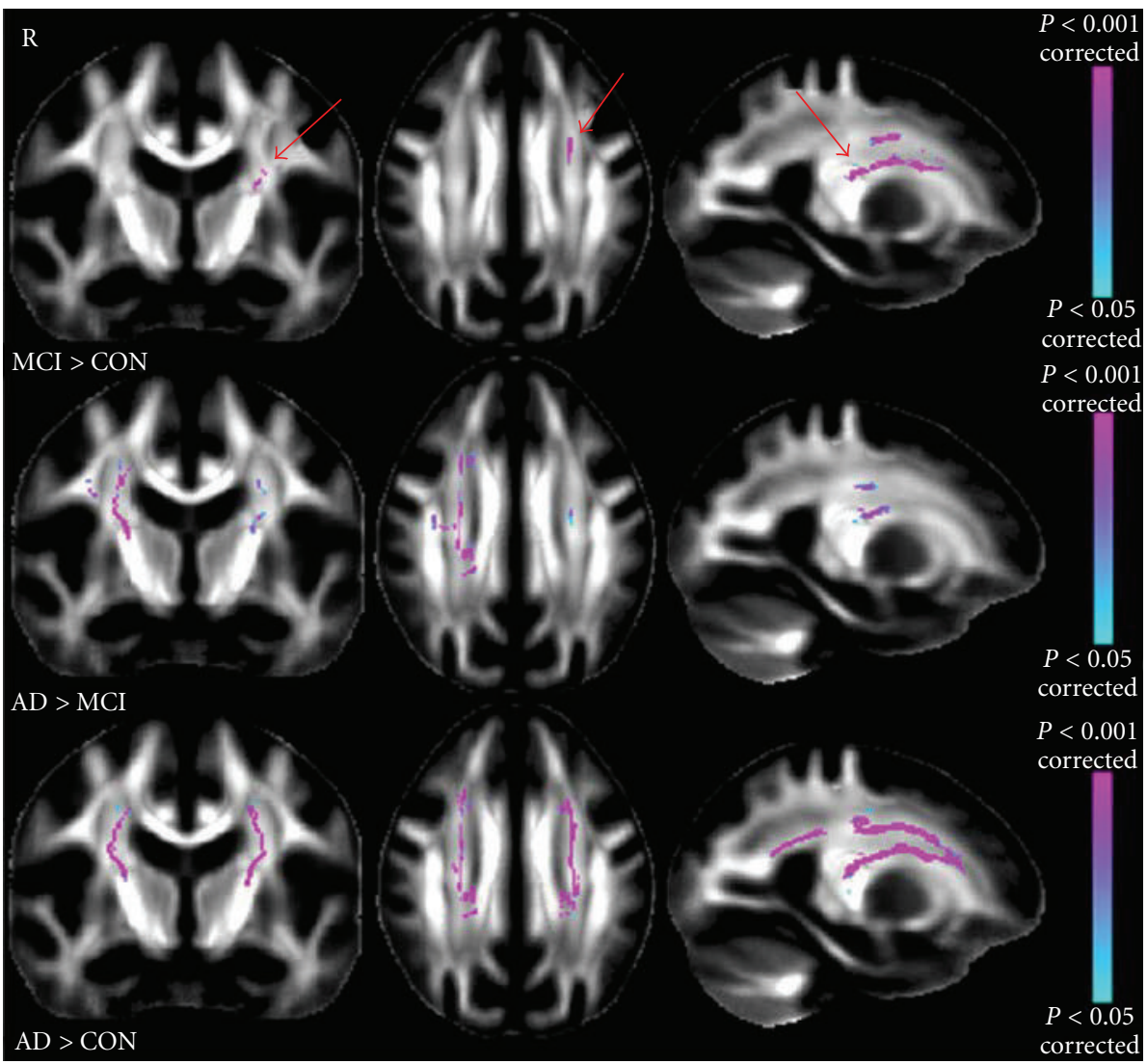

Figure 7: Top. Significant mode of atrophy (MO) results showing the contrast MCI > CON (controls; arrows). Middle. Significant MO results showing the contrast AD > MCI. Bottom. Significant MO results showing the contrast AD > CON. (adapted with permission from [34]).

with typical aging, but who do not show other symptoms of dementia, such as impaired judgment or reasoning. MCI has various clinical subtypes, including amnestic single domain (aMCI-S), amnestic multiple domain (aMCI-M), nonamnestic single domain (naMCI-S), and nonamnestic multiple domain (naMCI-M) [48]. Nonamnestic forms of MCI (naMCI, i.e., naMCI-S and naMCI-M) have had findings suggestive of vascular disease, whereas amnestic forms of MCI (aMCI, i.e., aMCI-S and aMCI-M) have appeared to have demographic, genetic, and MRI findings suggestive of AD pathology [48,49]. Although aMCI can be defined using neuropsychiatric criteria, brain imaging studies have aimed to develop measures that are sensitive enough to distinguish aMCI from normal aging with high specificity [50]. Many other studies attempt to differentiate between aMCI subjects who will convert to AD, over a specific followup interval versus those who remain stable or ever recover [51]. In this section, we elaborate the current findings in these two areas.

4.1. Distinguishing aMCI from Normal Aging. Although agerelated regional volume loss is apparent and widespread in nondemented individuals $[5,52]$, aMCI is associated with a unique pattern of structural vulnerability reflected in differential volume loss in specific regions. In a cross-sectional study, aMCI patients were observed with a significant WM abnormality in the region of crossing fibers in the centrum semiovale in comparison to NC (Figure 7) [34]. In a tenconsecutive-year longitudinal study, 18 participants (among 138) who converted from normal to MCI showed accelerated changes (compared to normal controls) on whole brain volume, ventricular CSF (vCSF), temporal gray matter, and orbitofrontal and temporal association cortices, including the hippocampus $(P \leq 0.04)$ (Figure 8) [35].

Similar findings of vCSF increases in aMCI patients compared to normal controls have been reported by Vemuri et al. [53]. In this study, Vemuri and colleagues further demonstrated that changes in serial structural MRI differed by $\mathrm{APOE} \varepsilon 4$ status overall among aMCI, with higher brain atrophy rates in APOE $\varepsilon 4$ carriers. In addition, MR-based structural biomarkers, compared with other biomarkers (e.g., CSF), showed higher correlation with concurrent change on general cognitive and functional indices in impaired subjects.

Functional abnormality has also been shown in aMCI patients. Compared with healthy controls, aMCI patients had a regional pattern of brain disconnection between the posterior cingulate cortex (PCC) and the medial prefrontal cortex and the rest of the brain. These disconnections 


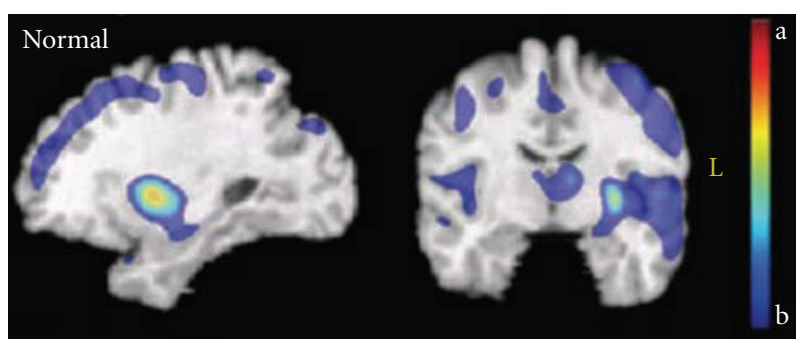

(a)

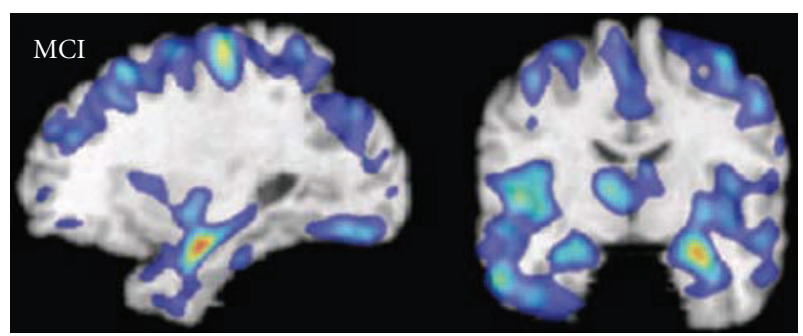

(b)

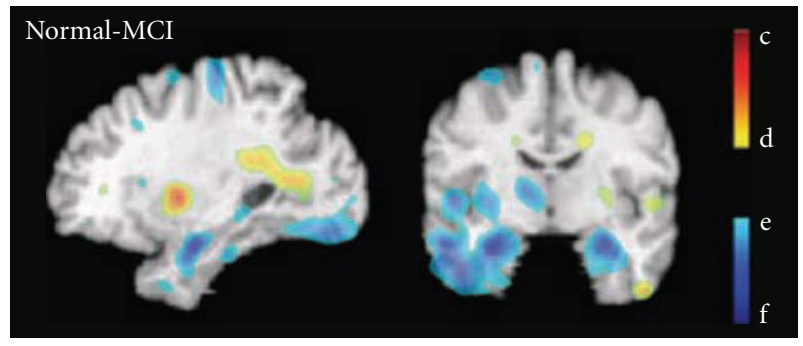

(c)

Figure 8: Patterns of GMV loss in MCI and normal aging. Average slopes of RAVENS maps for normal (a) and MCI (b) groups. The red-yellow color indicates greater volume loss. Bottom row: difference between the two groups; blue/green are regions in which MCI subjects showed higher rate of gray matter decrease. (c) Red/yellow colors reflect an increase of periventricular small vessel disease, which appears gray in T1-weighted images and is segmented as gray matter. The color bars display estimated regression coefficients and are defined by the following numbers, all in $\mathrm{mm}^{3}$ /year (per voxel in the template space): $\mathrm{a}=-0.020$, $\mathrm{b}=-0.0053, \mathrm{c}=0.026, \mathrm{~d}=0.0053, \mathrm{e}=-0.0053$, and $\mathrm{f}=-0.023$ (adapted with permission from [35]).

could be observed even in the absence of GM atrophy (Figure 9) [36].

4.2. Conversion from aMCI to $A D$. Cognitively normal elderly subjects convert to $\mathrm{AD}$ at a rate of only $1-2 \%$ per year, whereas aMCI subjects convert to $\mathrm{AD}$ at a rate of $12-15 \%$ per year [54]. Studying the similarities and differences between aMCI and $\mathrm{AD}$ would provide valuable information of the disease mechanism and progression. Multimodal MRI offers noninvasive methods for detection and possibly prediction of the conversion from aMCI to $\mathrm{AD}$ [6-8]. In a 3-year followup of 118 aMCI individuals who progressed to a diagnosis of $\mathrm{AD}$, Desikan et al. reported that atrophy in the medial temporal cortex (as measured by hippocampal volume, entorhinal cortex thickness, amygdala volume, temporal pole thickness, and parahippocampal gyrus thickness) can accurately and reliably predict time to disease progression $[6,7]$. They demonstrated that aMCI individuals with significant atrophy of the medial temporal factor regions are three times as likely to progress to AD, compared with aMCI individuals with preserved medial temporal factor regions. Their results also demonstrated that amygdala and temporal pole may be additional important structures for predicting the conversion from aMCI to AD. Similar observations were found in a meta-analysis involving 40 studies of imaging data from 1351 patients, suggesting that atrophy in the (trans) entorhinal area and hippocampus most reliably predict the progression from aMCI to $\mathrm{AD}$ [55]. These data provide strong evidence that $\mathrm{AD}$-related volume losses are most readily detected in the medial temporal lobe in aMCI. The reduction in medial temporal lobe volume is therefore an important indicator in predicting the transition of aMCI to AD.

MR-based ASL techniques provide a functional biomarker (perfusion) to predict the progression from MRI to $\mathrm{AD}$. In a longitudinal study $(2.7 \pm 1.0$ years $)$, Chao et al. reported that the MCI individuals who converted to dementia displayed hypoperfusion in the right precuneus, right inferior parietal cortex, and right middle frontal cortex [56]. A similar finding was reported in the Schroeter et al. meta-analytic study (involving 1351 patients) in which hypoperfusion in the inferior parietal lobules was found to most reliably predict the progression from aMCI to AD [55]. Furthermore, baseline perfusion from the right precuneus predicted subsequent declines in clinical dementia rating sum of boxes, functional activates questionnaire and selective attention (Stroop Switching), and baseline perfusion from the right middle frontal cortex predicted subsequent episodic memory decline. These results suggest that hypoperfusion as detected by ASL MRI can predict progression from MCI to AD.

\section{Alzheimer's Disease}

$\mathrm{AD}$ is histopathologically characterized by the formation of $\beta$-amyloid $(\mathrm{A} \beta)$ plaques and neurofibrillary tangles (NFT). Progressions of the $A \beta$ plaques and NFT pathology of AD correlate closely with loss of neurons and synapses [57]. These losses further result in gross atrophy, including cortical gray matter loss, reduced subcortical gray, and white matter volumes, as well as expanding ventricular and sulcal cerebrospinal fluid (CSF) spaces $[37,38]$ (Figure 10; similar regions of $\mathrm{A} \beta / \mathrm{NTF}$ deposition and brain atrophy in $\mathrm{AD})$. In $\mathrm{AD}$, this brain atrophy is localized to the medial temporal limbic cortex during its earliest states. At later stages of disease, it progresses to paralimbic cortical regions and the neocortex [57]. The temporal limbic cortex has essential roles in episodic memory. Since memory impairment is the earliest symptom of $\mathrm{AD}$, the temporal limbic cortex (including entorhinal cortex and hippocampus) has been an attractive target for structural neuroimaging studies [58-62].

Using brain volumetric measurements, patients with mild AD showed significantly smaller brain regions of 


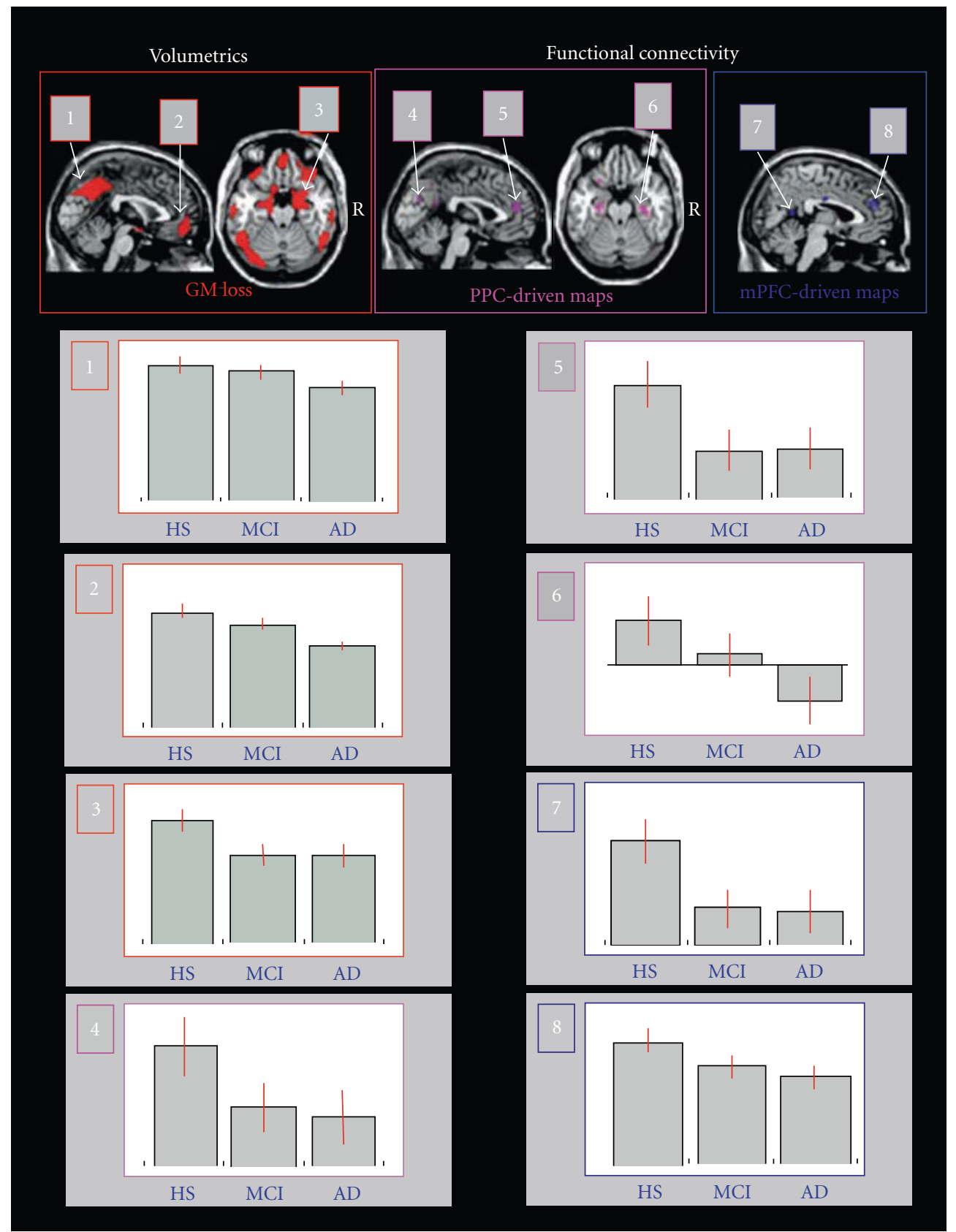

FIGURE 9: Distribution of reduced regional GMV (top panel on the left) and functional connectivity (top panel on the right) observed in patients with fully developed dementia (AD) compared with healthy subjects (HS). Changes of functional connectivity were assessed using both posterior cingulate (PCC) and medial prefrontal cortex (mPFC) driven connectivity maps (adapted with permission from [36]).

hippocampus (25\%) and entorhinal cortex (37\%) than healthy elderly controls [58-62]. In a number of longitudinal studies, significantly higher rates of brain atrophy were observed in AD [63-66]. The global atrophy rate in normal aging typically increases from $0.3 \%$ to $0.5 \%$ per year at age $70-80$ but increases from $2 \%$ to $3 \%$ per year in AD [6769]. Similar regional observations have also been found in hippocampus (controls, $1.0 \%$ to $1.7 \%$ per year; $\mathrm{AD}, 3.0 \%$ to $5.9 \%$ per year) and in entorhinal cortex (controls, $1.4 \%$ to $2.9 \%$ per year; $\mathrm{AD}, 7.15$ to $8.4 \%$ per year) $[2,70,71]$.
MR-based volume measures, particularly for the hippocampus, have been shown to be a strong structural biomarker for $\mathrm{AD}$, as follows [72-74]. First, it has been demonstrated that a significant correlation exists between MRI and histological-based hippocampal volumes $(r=0.97$, $P<0.001)$; the difference in the hippocampal volumes between normal and AD groups was $42 \%$ for the MRI data, and $40 \%$ for the histology data after adjusting for tissue shrinkage during specimen processing. Moreover, both the histological and MRI hippocampal volume measurements 


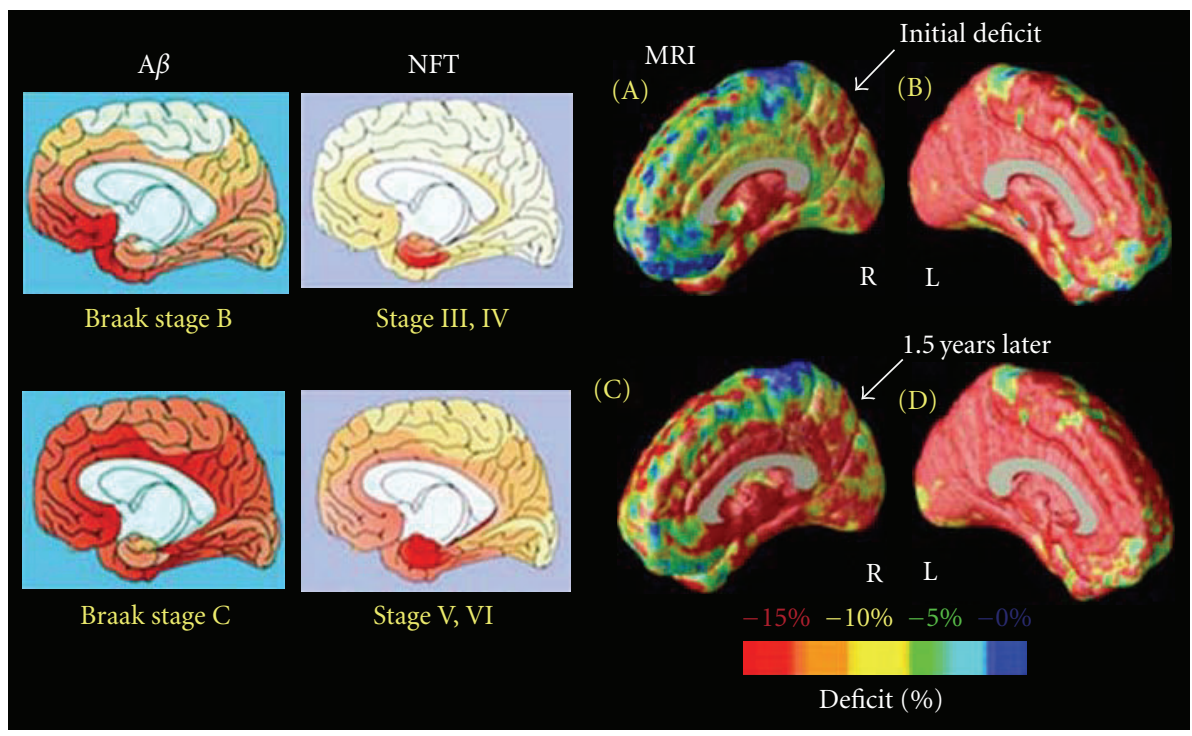

FIGURE 10: Gray matter deficits spread through the limbic system in moderate AD. Average maps of gray matter density in patients and controls were subtracted at their first scan (A and B) and at their followup scan 1.5 years later (C and D). Colors show the average percentage loss of gray matter relative to the control average. Profound loss engulfs the left medial wall (>15\%; B and D). On the right, however, the deficits in temporoparietal and entorhinal territory (A) spread forward into the cingulate gyrus 1.5 years later (C). Limbic and frontal zones clearly show different degrees of impairment (C). MRI-based changes, in living patients, agree strongly with the spatial progression of $\beta$-amyloid $(\mathrm{A} \beta)$ and NFT pathology observed after mortem (Braak Stages B, C, and III to VI), (adapted with permission from [37, 38]).

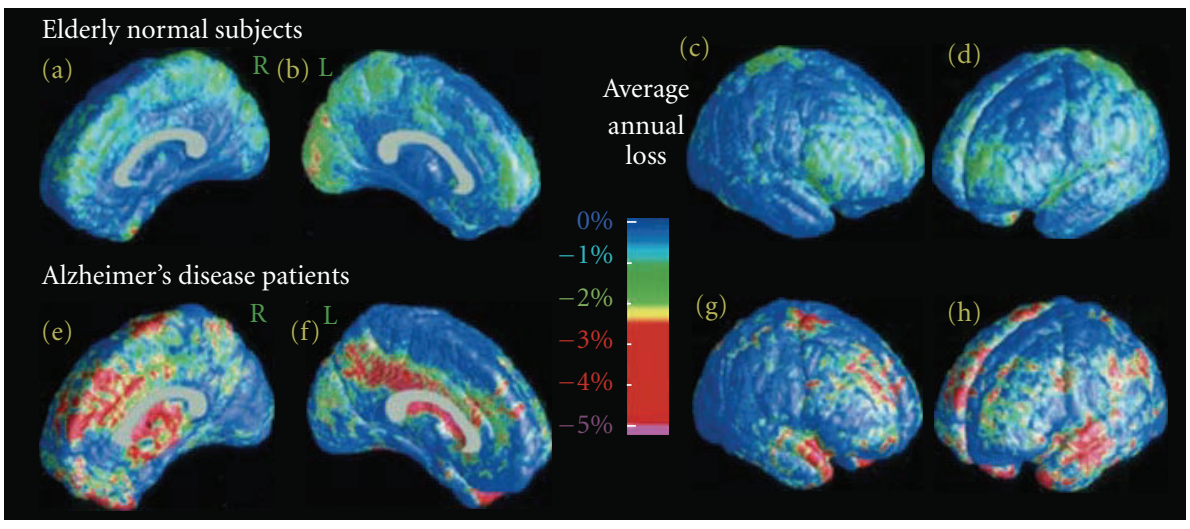

FIGURE 11: Average gray matter loss rates in healthy aging and AD. The maps show the average local rates of loss for gray matter, in groups of controls (top, (a)-(d)) and patients with AD (bottom, (e)-(h)). Loss rates are $<1 \%$ per year in controls. They are significantly higher in $\mathrm{AD}$ and strongest in frontal and temporal regions $(\mathrm{g}, \mathrm{h})$ at this stage of $\mathrm{AD}$. (adapted with permission from [39]).

were significantly associated with the number of hippocampal neurons $(r=0.91, P<0.001$ and $r=0.90, P<0.01)$. Second, when compared with a temporal lobe neocortical reference volume, the hippocampal volume showed an anatomically unique correlation to memory performance such as delayed verbal recall [72-74].

With GMT measurements, mild-to-moderate AD subjects have cortices that are an average of $18 \%$ thinner relative to healthy controls $(\mathrm{AD}=3.1 \pm 0.28 \mathrm{~mm}$, controls $=3.74 \pm$ $0.32 \mathrm{~mm}$ ) [75]. Significant GMT declines in AD were found in temporal, orbitofrontal, and parietal regions. The most pronounced changes occur in the allocortical region of the medial temporal lobes, which outlines the parahippocampal gyrus representing a loss of $>1.25$ millimeters of cortical thickness $[75,76]$. In a followup study 1.5 years later, patients with $\mathrm{AD}$ lost significant GM $(P<0.05$ for overall annual loss of gray matter) (Figures $11(\mathrm{e})-11(\mathrm{~h})$; [39]) at a significantly higher rate than controls $(P<0.042)$, with a total gray matter loss rate of $5.03 \pm 2.28 \%$ per year (left hemisphere $5.43 \pm 3.29 \%$ per year; right hemisphere $4.64 \pm 3.31 \%$ per year, whereas few regions in healthy controls exceeded a $1 \%$ annual gray matter loss). Regions with a prominent 4$5 \%$ annual loss included the right cingulate, temporal, and frontal cortices bilaterally (Figure 11, bottom row).

GMT changes are strong structural biomarkers for AD. Highly significant linkage was found relating greater GMT deficits to lower cognitive scores on the MMSE (Figure 12; [39]). These correlations were observed in all 


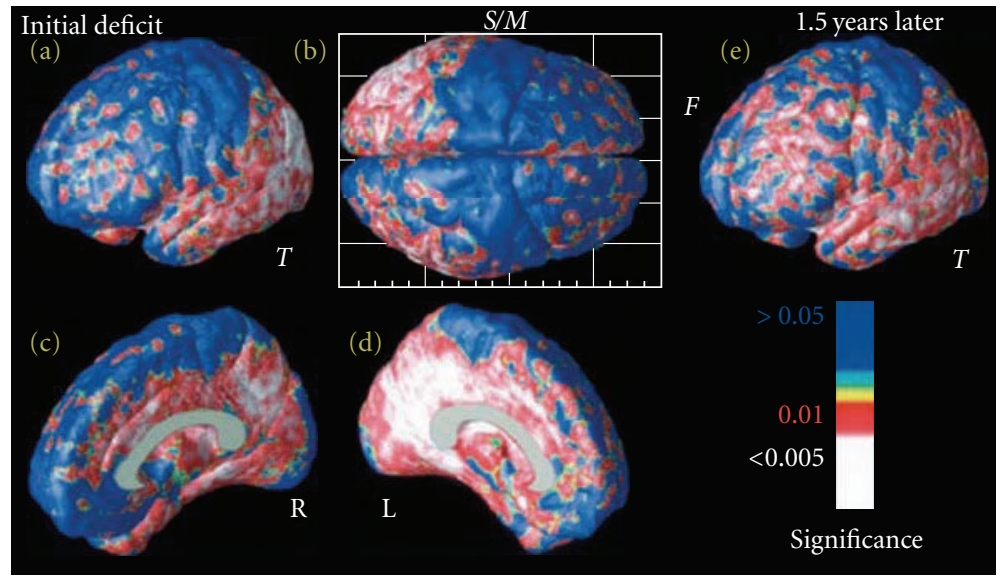

FIGURE 12: Mapping links between cognitive performance and changing brain structure. These maps show the significance of the linkage between gray matter reductions and cognition, as measured by MMSE score. Variations in temporal, parietal, and ultimately frontal (e) tissue are linked with cognitive status. Less gray matter is strongly correlated with worse cognitive performance, in all regions with prominent deficits. Linkages are detected most strongly in the left hemisphere medial temporoparietal zones (d). As expected, no linkages are found with sensorimotor gray matter variation (b), which was not in significant deficit in late AD (adapted with permission from [39]).

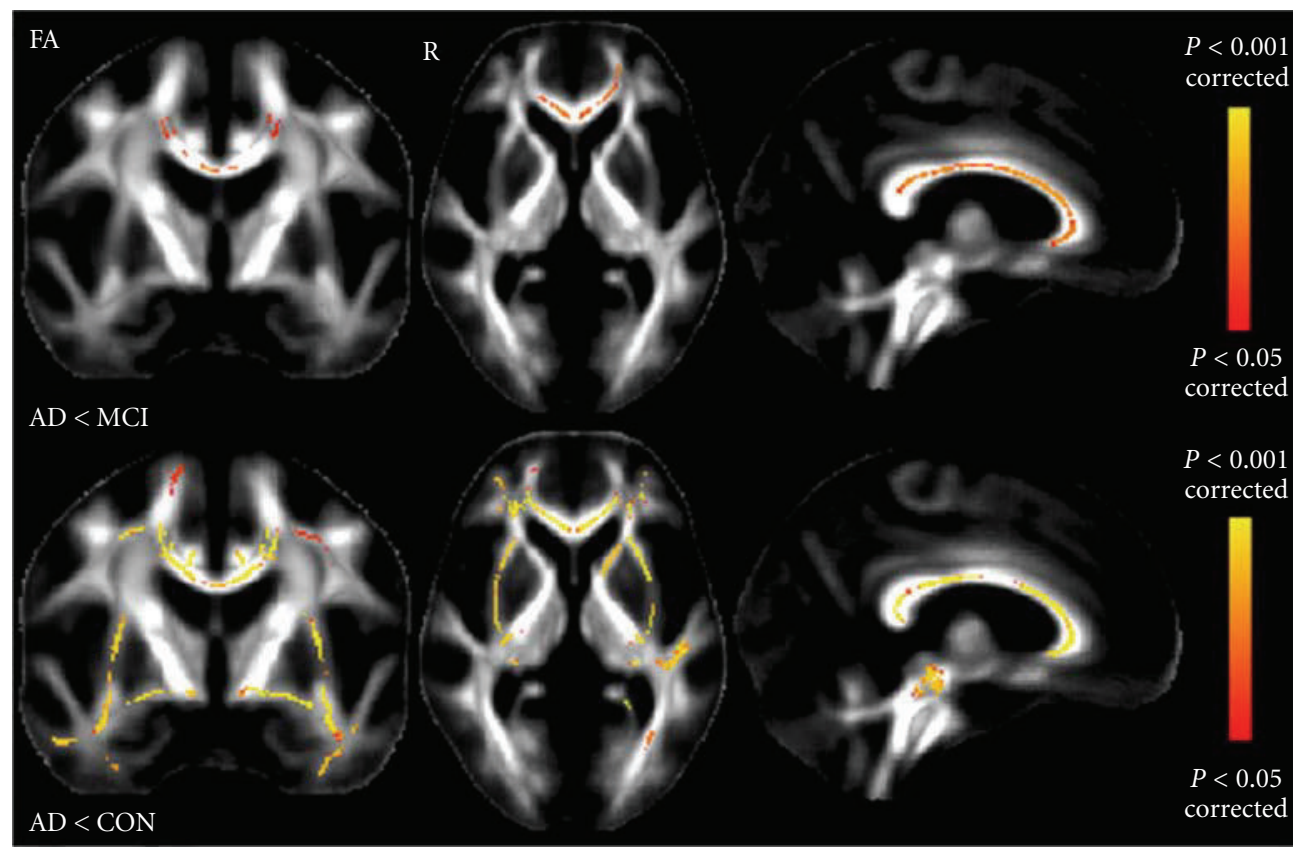

FIGURE 13: First row: significant FA results showing the contrast MCI > AD; second row: significant FA results showing CON (controls) > $\mathrm{AD}$ (adapted with permission from [34]).

brain regions, including the temporal, parietal, and limbic cortices. Correlations were also found between frontal gray matter reduction and lower MMSE scores, but only at the later time point, when frontal gray matter was in significant deficit (Figure 12(e)). No correlations were found between gray matter differences in sensory and motor cortices and cognitive performance (Figure 12(b), blue, S/M). These results support the theory that the relationship between brain structure and cognition is regionally specific in $\mathrm{AD}$, at least initially (Figure 12(b)). Correlations were observed to be strongest in regions with greatest average loss, such as the left cingulate and left temporal and parietal cortices (Figure 12(d)).

WM degeneration has also been considered an important indicator of $\mathrm{AD}$. In a comparison of healthy young versus older adults, WM has been found to decline in volume with increasing age but is further reduced in $\mathrm{AD}$, with parahippocampal, entorhinal, inferior parietal, and rostral 

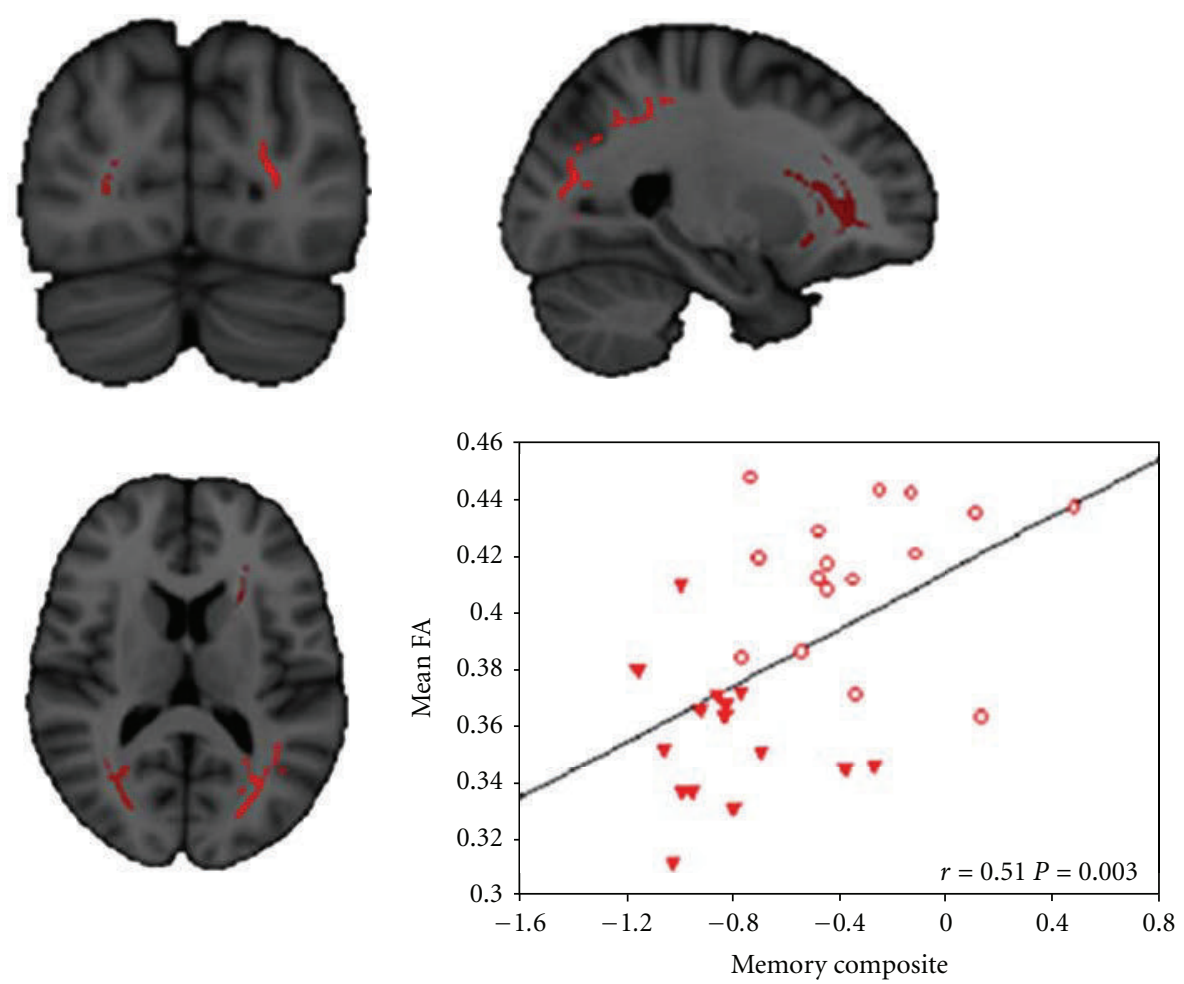

FIGURE 14: Significant correlations between regional alterations of FA in patients and the composite memory scores (adapted with permission from [40]).

middle frontal areas showing the strongest $\mathrm{AD}$-associated reductions in WM [77]. AD patients, similar to aMCI patients (but more severe), have shown significant increase in diffusion atrophy in the region of crossing fibers in the centrum semiovale (Figure 6; [34]), They have further shown regionally specific shape abnormalities and reduction in fractional anisotropy (FA) in the corpus callosum, anterior commissure, uncinate fasciculus, cingulum tract, and sagittal stratum tract (these have not observed in aMCI patients in comparison to NC; Figure 13; $[34,78,79])$. These results suggest that disruption in the white matter tracts near the temporal lobe may represent the secondary consequence of the medial temporal lobe pathology in AD. This is consistent with the observation that FA values are significantly related to memory performance among AD patients (Figure 14 ; [40]).

Structural imaging is able to detect AD only at a stage in which the disease has progressed so far that neurons are already irreversibly lost. Ideally, AD should be diagnosed at an earlier stage in which neurons are impaired by the disease process, but not yet fully damaged, and thus can be potentially salvaged $[2,13]$. In contrast, alterations of neuronal activity, metabolism, and hemodynamics are accompanied by the impairment of neurons and usually precede neuronal death prior to any cognitive deficits. Functional MRI has shown great promise in the detection of $\mathrm{AD}$ at this very early stage of disease, as well as during disease progression.

Disrupted functional connectivity has been observed in patients with $\mathrm{AD}$ in the default mode network, which is associated with autobiographical memory retrieval [80, 81]. Similar to aMCI, functional connectivity abnormalities were observed in the PCC and a set of default mode regions, including the medial prefrontal cortex, hippocampus, inferior temporal cortex, bilateral visual cortices, and the precuneus in AD patients [82]. This disruption of connectivity was observed to intensify during aMCI and AD disease progression (Figure 15; [41]). With concurrent fcMRI and structural MRI measurements, the PCC showed reduced connectivity in patients progressing into $\mathrm{AD}$ even in the absence of GM atrophy (Figure 9; [36]). This indicates that functional connectivity abnormalities precede GM atrophy in the PCC and supports the hypothesis that GM atrophy in specific regions of AD brains likely reflects a long-term effect of brain disconnection.

In $\mathrm{AD}$ patients, significant declines of $\mathrm{CBF}$ have been found in frontal, parietal, and temporal regions $(P<$ 0.001), with more marked reductions in those patients with severe dementia. Covariance analysis revealed that aging and disease severity have a pronounced effect on CBF, especially that of the left parietal region. Significant decreases of $\mathrm{CBF}$ have been detected with ASL-MRI in temporal, parietal, and frontal cortex and the posterior cingulate in patients with $\mathrm{AD}$, compared to the healthy elderly controls. The observations have been consistent with those observed using SPECT. Using dynamic contrast method, CBF has been found significantly reduced in insular cortex $[83,84]$. Because the insula is an important brain structure for the 

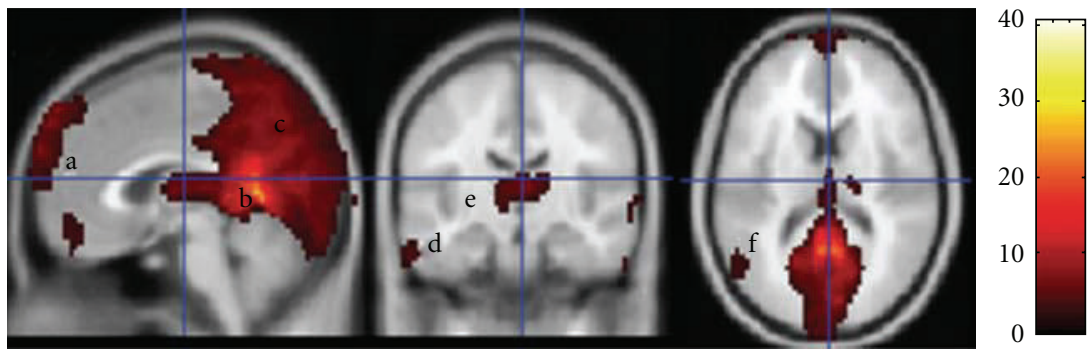

(a)
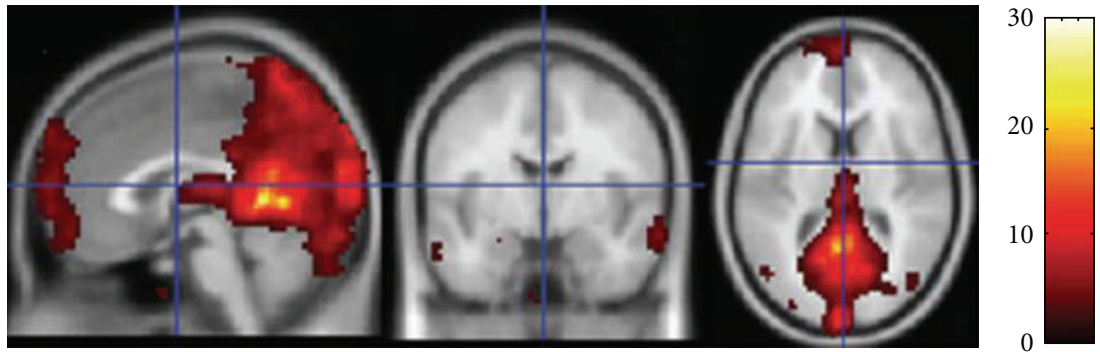

(b)
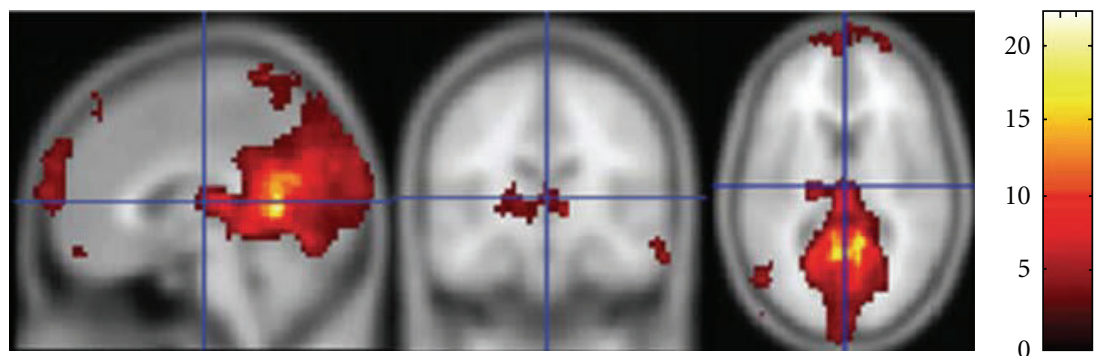

(c)
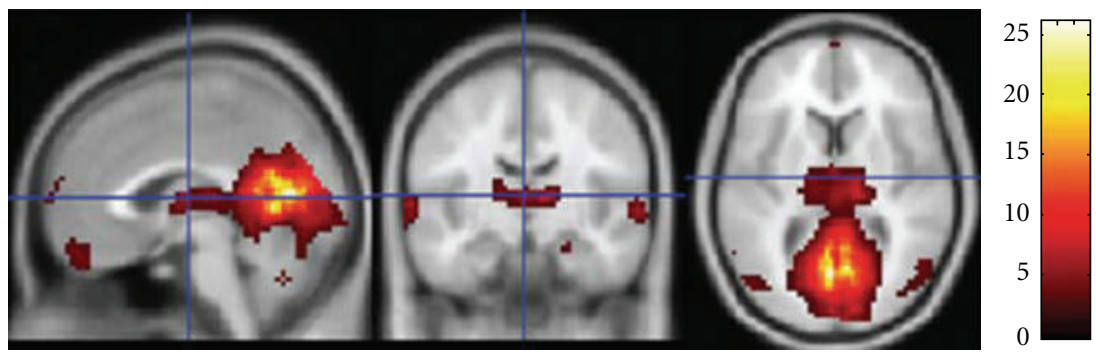

(d)

FIGURE 15: (a-d) Left to right: sagittal, coronal, and axial views of T1-weighted MNI canonical brain templates show intragroup maps of the default mode network (DMN) based on the seed of the PCC in, (a), control group, (b), mild AD group, (c), moderate AD group, and (d), severe AD group. The regions involved in the DMN are labeled as follows in (a): $a=$ ventral MPFC, $b=$ PCC, $c=$ precuneus and/or cuneus, $\mathrm{d}=\mathrm{ITC}, \mathrm{e}=$ thalamus, and $\mathrm{f}=$ inferior parietal cortex. Color scale $=t$ values (adapted with permission from [41]).

autonomic control of blood pressure and heart rate, the observations suggested that AD pathology has effect on ventral autonomic cardioregulatory dysfunction.

In $\mathrm{AD}$ patients, significant $\mathrm{N}$-acetyl aspartate (NAA) reductions have been found in various brain areas, including PCC, hippocampus, and GM of the temporal, parietal, and sometimes the frontal as well as occipital cortices $[23,85]$. Decrease in NAA reflects a combination of losses of neuronal cells/dendritic structures, reduced myelination, and decreased neuronal metabolism. As a result, the degree of cognitive impairment has been well correlated with the degree of NAA decrease; poor performance on memory tests correlated with lower gray matter NAA level [86]. In contrast to NAA, myoinositol (MI), a glial biomarker, has been found to be dramatically increased in $\mathrm{AD}$. Elevated $\mathrm{MI}$ is most likely due to the increase of gliosis in AD. Taken together, NAA and MI are important and useful metabolic biomarkers to distinguish $\mathrm{AD}$ from normal aging. 


\section{Future Directions}

Mitochondrial dysfunction is a well-known biomarker of AD. Mitochondria are the predominate source $(>98 \%)$ of energy production in mammals, yielding ATP through oxidative phosphorylation of glucose. In the brain, oxidative metabolism $\left(\mathrm{O}_{2}\right.$ consumption) is the predominant source of energy (ATP generation), supporting baseline demands and maintaining viability, as well as responding rapidly and in a highly regional manner to changes in neuronal activity induced by task performance. Failure to maintain adequate levels of tissue oxygenation rapidly results in tissue death as observed of brain atrophy in $\mathrm{AD}$ patients.

To identify the integrity of the mitochondrial function, cerebral metabolic rate of glucose $\left(\mathrm{CMR}_{\mathrm{Glc}}\right)$ and oxygen $\left(\mathrm{CMRO}_{2}\right)$ are the most-well known indicators [87]. CMR $\mathrm{Glc}$ measurements in $\mathrm{AD}$ research have been well established with positron emission tomography (PET) methods. For instance, significant decreases of glucose metabolism have been found in young APOE $\varepsilon 4$ carriers (30 years old) in brain areas associated with $\mathrm{AD}$ pathology [88]. In contrast, $\mathrm{CMRO}_{2}$ measurements are not feasible using PET methods, especially for $\mathrm{AD}$ patients, due to the difficulties of obtaining arterial blood samples. In addition, the radioactive nature of PET allows less repetitive scans, which limits the monitoring of the disease progress. Therefore, considerable efforts have been made to develop MRI-based, noninvasive, $\mathrm{CMRO}_{2}$ measurements. Baseline $\mathrm{CMRO}_{2}$ and task-induced changes in $\mathrm{CMRO}_{2}$ determinations have been proposed by several methods, including $\mathrm{T}_{2}$-relaxation-under-spin-tagging (TRUST) and quantitative BOLD (qBOLD) techniques [89-93]. These MR-based metabolic imaging methods, in addition to MRS, are expected to be very useful as diagnostic and prognostic biomarkers in AD. However, future studies allowing for rigorous assessment of test-retest reliability and power calculation compared to the established imaging techniques are necessary before these $\mathrm{CMRO}_{2}$ methods can be accepted as other complementary and/or established functional neuroimaging biomarkers for $\mathrm{AD}$.

The development and validation of structural and functional biomarkers will enable MRI to be utilized as a powerful tool for evaluation of therapeutic efficacy in AD in largescale clinical trials. For example, Jack et al. estimated that in each arm of a therapeutic trail with conventional volumetric measures for hippocampal volume, only 21 subjects would be required to detect $50 \%$ reduction in the rate of decline. This compares 241 subjects if MMSE scores were used; 320 subjects if ADAS Cog scores were used [54]. Combining with other biomarkers (e.g., $\mathrm{CMR}_{\mathrm{Glc}}$ by PET and A $\beta / \mathrm{NFT}$ CSF), surrogate markers for $\mathrm{AD}$ progress can be identified and used for clinical/cognitive tests in clinical trials. Nonetheless, these surrogate markers must be validated to be reproducible in the treatment setting, across various types of treatments, across imaging centers, and across time. A multicenter $\mathrm{AD}$ research project, known as the Alzheimer's Disease Neuroimaging Initiative (ANDI) (http://adni.loni.ucla.edu/), was launched in 2004 to meet this goal.

\section{Conclusions}

The incidence of Alzheimer's disease is increasing with the extended lifespan in developed countries. The development of neuroimaging biomarkers is a pressing need to detect the early risk of $\mathrm{AD}$ (from NC), predict and monitor disease progression (from aMCI). Multimodal MRI methods have been developed to meet this demand by providing useful and important structural and functional biomarkers in $\mathrm{AD}$. The validation of the surrogate biomarkers will have profound implications in $\mathrm{AD}$ clinical trials, including the prevention and deceleration of $\mathrm{AD}$ onset, as well as the evaluation of treatment efficacy.

\section{References}

[1] L. E. Hebert, P. A. Scherr, J. L. Bienias, D. A. Bennett, and D. A. Evans, "Alzheimer disease in the US population: prevalence estimates using the 2000 census," Archives of Neurology, vol. 60, no. 8, pp. 1119-1122, 2003.

[2] S. G. Mueller, M. W. Weiner, L. J. Thal et al., "Ways toward an early diagnosis in Alzheimer's disease: the Alzheimer's disease neuroimaging initiative (ADNI)," Alzheimer's and Dementia, vol. 1, no. 1, pp. 55-66, 2005.

[3] R. C. Mohs, D. Marin, and V. Haroutunian, "Early clinical and biological manifestations of Alzheimer's disease: implications for screening and treatment," in Drug Discovery and Development for Alzheimer's Disease, H. Fillit and A. W. O'Connell, Eds., Springer, New York, NY, USA, 2002.

[4] G. B. Frisoni, "Structural imaging in the clinical diagnosis of Alzheimer's disease: problems and tools," Journal of Neurology Neurosurgery and Psychiatry, vol. 70, no. 6, pp. 711-718, 2001.

[5] P. Kochunov, J. F. Mangin, T. Coyle et al., "Age-related morphology trends of cortical sulci," Human Brain Mapping, vol. 26, no. 3, pp. 210-220, 2005.

[6] R. S. Desikan, H. J. Cabral, F. Settecase et al., "Automated MRI measures predict progression to Alzheimer's disease," Neurobiology of Aging, vol. 31, no. 8, pp. 1364-1374, 2010.

[7] R. S. Desikan, H. J. Cabral, C. P. Hess et al., "Automated MRI measures identify individuals with mild cognitive impairment and Alzheimers disease," Brain, vol. 132, no. 8, pp. 2048-2057, 2009.

[8] W. Yang, R. L.M. Lui, J. -H. Gao et al., "Independent component analysis-based classification of Alzheimer's disease MRI data," Journal of Alzheimer's Disease, vol. 24, no. 4, pp. 775-783, 2011.

[9] P. Kochunov, P. M. Thompson, J. L. Lancaster et al., "Relationship between white matter fractional anisotropy and other indices of cerebral health in normal aging: tract-based spatial statistics study of aging," NeuroImage, vol. 35, no. 2, pp. 478487, 2007.

[10] P. Kochunov, D. E. Williamson, J. Lancaster et al., "Fractional anisotropy of water diffusion in cerebral white matter across the lifespan," Neurobiology of Aging. In press.

[11] B. Biswal, F. Z. Yetkin, V. M. Haughton, and J. S. Hyde, "Functional connectivity in the motor cortex of resting human brain using echo-planar MRI," Magnetic Resonance in Medicine, vol. 34, no. 4, pp. 537-541, 1995.

[12] D. Cordes, V. M. Haughton, K. Arfanakis et al., "Mapping functionally related regions of brain with functional connectivity MR imaging," American Journal of Neuroradiology, vol. 21, no. 9, pp. 1636-1644, 2000. 
[13] S. I. Rapoport, "Coupled reductions in brain oxidative phosphorylation and synaptic function can be quantified and staged in the course of Alzheimer disease," Neurotoxicity Research, vol. 5, no. 6, pp. 385-397, 2003.

[14] J. Xiong, L. M. Parsons, J. H. Gao, and P. T. Fox, "Interregional connectivity to primary motor cortex revealed using MRI resting state images," Human Brain Mapping, vol. 8, no. 2-3, pp. 151-156, 1999.

[15] C. F. Beckmann, M. DeLuca, J. T. Devlin, and S. M. Smith, "Investigations into resting-state connectivity using independent component analysis," Philosophical Transactions of the Royal Society B, vol. 360, no. 1457, pp. 1001-1013, 2005.

[16] J. S. Damoiseaux, S. A. R. B. Rombouts, F. Barkhof et al., "Consistent resting-state networks across healthy subjects," Proceedings of the National Academy of Sciences of the United States of America, vol. 103, no. 37, pp. 13848-13853, 2006.

[17] V. Kiviniemi, J. H. Kantola, J. Jauhiainen, A. Hyvärinen, and O. Tervonen, "Independent component analysis of nondeterministic fMRI signal sources," NeuroImage, vol. 19, no. 2, pp. 253-260, 2003.

[18] J. A. Detre, J. S. Leigh, D. S. Williams, and A. P. Koretsky, "Perfusion imaging," Magnetic Resonance in Medicine, vol. 23, no. 1, pp. 37-45, 1992.

[19] H. L. Liu, Y. Pu, Y. Liu et al., "Cerebral blood flow measurement by dynamic contrast MRI using singular value decomposition with an adaptive threshold," Magnetic Resonance in Medicine, vol. 42, no. 1, pp. 167-172, 1999.

[20] W. J. Jagust, "Functional imaging patterns in Alzheimer's disease: relationships to neurobiology," Annals of the New York Academy of Sciences, vol. 777, pp. 30-36, 1996.

[21] G. Waldemar, "Functional brain imaging with SPECT in normal aging and dementia: methodological, pathophysiological, and diagnostic aspects," Cerebrovascular and Brain Metabolism Reviews, vol. 7, no. 2, pp. 89-130, 1995.

[22] M. L. Simmons, C. G. Frondoza, and J. T. Coyle, "Immunocytochemical localization of $\mathrm{N}$-acetyl-aspartate with monoclonal antibodies," Neuroscience, vol. 45, no. 1, pp. 37-45, 1991.

[23] K. Kantarci, R. C. Petersen, B. F. Boeve et al., "1H MR spectroscopy in common dementias," Neurology, vol. 63, no. 8, pp. 1393-1398, 2004.

[24] E. Gómez-Tortosa, M. S. Barquero, M. Barón et al., "Variability of age at onset in siblings with familial Alzheimer disease," Archives of Neurology, vol. 64, no. 12, pp. 1743-1748, 2007.

[25] L. Mosconi, R. Mistur, R. Switalski et al., "Declining brain glucose metabolism in normal individuals with a maternal history of Alzheimer disease," Neurology, vol. 72, no. 6, pp. 513-520, 2009.

[26] L. Mosconi, J. O. Rinne, W. H. Tsui et al., "Increased fibrillar amyloid- $\beta$ burden in normal individuals with a family history of late-onset Alzheimer's," Proceedings of the National Academy of Sciences of the United States of America, vol. 107, no. 13, pp. 5949-5954, 2010.

[27] R. A. Honea, R. H. Swerdlow, E. D. Vidoni, J. Goodwin, and J. M. Burns, "Reduced gray matter volume in normal adults with a maternal family history of Alzheimer disease," Neurology, vol. 74, no. 2, pp. 113-120, 2010.

[28] R. A. Honea, R. H. Swerdlow, E. D. Vidoni, and J. M. Burns, "Progressive regional atrophy in normal adults with a maternal history of Alzheimer disease," Neurology, vol. 76, no. 9, pp. 822-829, 2011.

[29] S. Debette, P. A. Wolf, A. Beiser et al., "Association of parental dementia with cognitive and brain MRI measures in middleaged adults," Neurology, vol. 73, no. 24, pp. 2071-2078, 2009.
[30] F. Crivello, H. Lemaitre, C. Dufouil et al., "Effects of ApoE$\varepsilon 4$ allele load and age on the rates of grey matter and hippocampal volumes loss in a longitudinal cohort of 1186 healthy elderly persons," NeuroImage, vol. 53, no. 3, pp. 10641069, 2010.

[31] N. Filippini, M. Zarei, C. F. Beckmann et al., "Regional atrophy of transcallosal prefrontal connections in cognitively normal APOE $\varepsilon 4$ carriers," Journal of Magnetic Resonance Imaging, vol. 29, no. 5, pp. 1021-1026, 2009.

[32] S. Y. Bookheimer, M. H. Strojwas, M. S. Cohen et al., "Patterns of brain activation in people at risk for Alzheimer's disease," New England Journal of Medicine, vol. 343, no. 7, pp. 450-456, 2000.

[33] N. Filippini, K. P. Ebmeier, B. J. MacIntosh et al., "Differential effects of the APOE genotype on brain function across the lifespan," NeuroImage, vol. 54, no. 1, pp. 602-610, 2011.

[34] G. Douaud, S. Jbabdi, T. E. J. Behrens et al., "DTI measures in crossing-fibre areas: increased diffusion anisotropy reveals early white matter alteration in MCI and mild Alzheimer's disease," NeuroImage, vol. 55, no. 3, pp. 880-890, 2011.

[35] I. Driscoll, C. Davatzikos, Y. An et al., "Longitudinal pattern of regional brain volume change differentiates normal aging from MCI," Neurology, vol. 72, no. 22, pp. 1906-1913, 2009.

[36] T. Gili, M. Cercignani, L. Serra et al., "Regional brain atrophy and functional disconnection across Alzheimer's disease evolution," Journal of Neurology, Neurosurgery and Psychiatry, vol. 82, no. 1, pp. 58-66, 2011.

[37] P. M. Thompson, K. M. Hayashi, R. A. Dutton et al., "Tracking Alzheimer's disease," Annals of the New York Academy of Sciences, vol. 1097, pp. 183-214, 2007.

[38] P. M. Thompson, K. M. Hayashi, G. I. De Zubicaray et al., "Mapping hippocampal and ventricular change in Alzheimer disease," NeuroImage, vol. 22, no. 4, pp. 1754-1766, 2004.

[39] P. M. Thompson, K. M. Hayashi, G. De Zubicaray et al., "Dynamics of gray matter loss in Alzheimer's disease," Journal of Neuroscience, vol. 23, no. 3, pp. 994-1005, 2003.

[40] B. Bosch, E. M. Arenaza-Urquijo, L. Rami et al., "Multiple DTI index analysis in normal aging, amnestic MCI and AD. Relationship with neuropsychological performance," Neurobiology of Aging. In press.

[41] H. Y. Zhang, S. J. Wang, B. Liu et al., "Resting brain connectivity: changes during the progress of Alzheimer disease," Radiology, vol. 256, no. 2, pp. 598-606, 2010.

[42] R. G. Morris and M. D. Kopelman, "The memory deficits in Alzheimer-type dementia: a review," Quarterly Journal of Experimental Psychology Section A, vol. 38, no. 4, pp. 575-602, 1986.

[43] R. J. Caselli, A. C. Dueck, D. Osborne et al., "Longitudinal modeling of age-related memory decline and the APOE $\varepsilon 4$ effect," New England Journal of Medicine, vol. 361, no. 3, pp. 255-263, 2009.

[44] I. J. Deary, M. C. Whiteman, A. Pattie et al., "Ageing: cognitive change and the APOE $\varepsilon 4$ allele," Nature, vol. 418, no. 6901, p. 932, 2002.

[45] N. A. Dennis, J. N. Browndyke, J. Stokes et al., "Temporal lobe functional activity and connectivity in young adult APOE $\varepsilon 4$ carriers," Alzheimer's and Dementia, vol. 6, no. 4, pp. 303-311, 2010.

[46] N. Filippini, B. J. MacIntosh, M. G. Hough et al., "Distinct patterns of brain activity in young carriers of the APOE- $\varepsilon 4$ allele," Proceedings of the National Academy of Sciences of the United States of America, vol. 106, no. 17, pp. 7209-7214, 2009.

[47] Y. I. Sheline, J. C. Morris, A. Z. Snyder et al., "APOE4 allele disrupts resting state fMRI connectivity in the absence 
of amyloid plaques or decreased CSF A $\beta 42$," Journal of Neuroscience, vol. 30, no. 50, pp. 17035-17040, 2010.

[48] B. Winblad, K. Palmer, M. Kivipelto et al., "Mild cognitive impairment-beyond controversies, towards a consensus: report of the international working group on mild cognitive impairment," Journal of Internal Medicine, vol. 256, no. 3, pp. 240-246, 2004.

[49] J. He, S. Farias, O. Martinez, B. Reed, D. Mungas, and C. DeCarli, "Differences in brain volume, hippocampal volume, cerebrovascular risk factors, and apolipoprotein E4 among mild cognitive impairment subtypes," Archives of Neurology, vol. 66, no. 11, pp. 1393-1399, 2009.

[50] J. T. Becker, S. W. Davis, K. M. Hayashi et al., "Three-dimensional patterns of hippocampal atrophy in mild cognitive impairment," Archives of Neurology, vol. 63, no. 1, pp. 97-101, 2006.

[51] L. G. Apostolova, C. A. Steiner, G. G. Akopyan et al., “Threedimensional gray matter atrophy mapping in mild cognitive impairment and mild Alzheimer disease," Archives of Neurology, vol. 64, no. 10, pp. 1489-1495, 2007.

[52] P. Kochunov, A. E. Ramage, J. L. Lancaster et al., "Loss of cerebral white matter structural integrity tracks the gray matter metabolic decline in normal aging," NeuroImage, vol. 45, no. 1, pp. 17-28, 2009.

[53] P. Vemuri, H. J. Wiste, S. D. Weigand et al., "Serial MRI and CSF biomarkers in normal aging, MCI, and AD," Neurology, vol. 75, no. 2, pp. 143-151, 2010.

[54] C. R. Jack, M. Slomkowski, S. Gracon et al., "MRI as a biomarker of disease progression in a therapeutic trial of milameline for AD," Neurology, vol. 60, no. 2, pp. 253-260, 2003.

[55] M. L. Schroeter, T. Stein, N. Maslowski, and J. Neumann, "Neural correlates of Alzheimer's disease and mild cognitive impairment: a systematic and quantitative meta-analysis involving 1351 patients," NeuroImage, vol. 47, no. 4, pp. 11961206, 2009.

[56] L. L. Chao, S. T. Buckley, J. Kornak et al., "ASL perfusion MRI predicts cognitive decline and conversion from MCI to dementia," Alzheimer Disease and Associated Disorders, vol. 24, no. 1, pp. 19-27, 2010.

[57] H. Braak and E. Braak, "Neuropathological stageing of Alzheimer-related changes," Acta Neuropathologica, vol. 82, no. 4, pp. 239-259, 1991.

[58] A. T. Du, N. Schuff, D. Amend et al., "Magnetic resonance imaging of the entorhinal cortex and hippocampus in mild cognitive impairment and Alzheimer's disease," Journal of Neurology Neurosurgery and Psychiatry, vol. 71, no. 4, pp. 441-447, 2001.

[59] D. J. A. Callen, S. E. Black, F. Gao, C. B. Caldwell, and J. P. Szalai, "Beyond the hippocampus: MRI volumetry confirms widespread limbic atrophy in AD," Neurology, vol. 57, no. 9, pp. 1669-1674, 2001.

[60] C. R. Jack Jr., R. C. Petersen, Y. C. Xu et al., "Medial temporal atrophy on MRI in normal aging and very mild Alzheimer's disease," Neurology, vol. 49, no. 3, pp. 786-794, 1997.

[61] Y. Xu, C. R. Jack, P. C. O’Brien et al., "Usefulness of MRI measures of entorhinal cortex versus hippocampus in $\mathrm{AD}$," Neurology, vol. 54, no. 9, pp. 1760-1767, 2000.

[62] S. De Santi, M. J. De Leon, H. Rusinek et al., "Hippocampal formation glucose metabolism and volume losses in MCI and AD," Neurobiology of Aging, vol. 22, no. 4, pp. 529-539, 2001.

[63] N. C. Fox, S. Cousens, R. Scahill, R. J. Harvey, and M. N. Rossor, "Using serial registered brain magnetic resonance imaging to measure disease progression in Alzheimer disease: power calculations and estimates of sample size to detect treatment effects," Archives of Neurology, vol. 57, no. 3, pp. 339-344, 2000.

[64] C. R. Jack Jr., R. C. Petersen, Y. Xu et al., "Rate of medial temporal lobe atrophy in typical aging and Alzheimer's disease," Neurology, vol. 51, no. 4, pp. 993-999, 1998.

[65] J. A. Kaye, T. Swihart, D. Howieson et al., "Volume loss of the hippocampus and temporal lobe in healthy elderly persons destined to develop dementia," Neurology, vol. 48, no. 5, pp. 1297-1304, 1997.

[66] M. P. Laakso, M. Lehtovirta, K. Partanen, P. J. Riekkinen, and H. Soininen, "Hippocampus in Alzheimer's disease: a 3-year follow-up MRI study," Biological Psychiatry, vol. 47, no. 6, pp. 557-561, 1999.

[67] S. M. Resnick, D. L. Pham, M. A. Kraut, A. B. Zonderman, and C. Davatzikos, "Longitudinal magnetic resonance imaging studies of older adults: a shrinking brain," Journal of Neuroscience, vol. 23, no. 8, pp. 3295-3301, 2003.

[68] N. C. Fox and J. M. Schott, "Imaging cerebral atrophy: normal ageing to Alzheimer's disease," The Lancet, vol. 363, no. 9406, pp. 392-394, 2004.

[69] J. L. Gunter, M. M. Shiung, A. Manduca, and C. R. Jack, "Methodological considerations for measuring rates of brain atrophy," Journal of Magnetic Resonance Imaging, vol. 18, no. 1, pp. 16-24, 2003.

[70] A. T. Du, N. Schuff, J. H. Kramer et al., "Higher atrophy rate of entorhinal cortex than hippocampus in AD," Neurology, vol. 62, no. 3, pp. 422-427, 2004.

[71] C. R. Jack Jr., M. M. Shiung, J. L. Gunter et al., “Comparison of different MRI brain athrophy rate measures with clinical disease progression in AD," Neurology, vol. 62, no. 4, pp. 591600, 2004.

[72] M. J. De Leon, S. Desanti, R. Zinkowski et al., "MRI and CSF studies in the early diagnosis of Alzheimer's disease," Journal of Internal Medicine, vol. 256, no. 3, pp. 205-223, 2004.

[73] A. Convit, M. J. De Leon, C. Tarshish et al., "Specific hippocampal volume reductions in individuals at risk for Alzheimer's disease," Neurobiology of Aging, vol. 18, no. 2, pp. 131-138, 1997.

[74] J. Golomb, A. Kluger, M. J. de Leon et al., "Hippocampal formation size in normal human aging: a correlate of delayed secondary memory performance," Learning Memory, vol. 1, no. 1, pp. 45-54, 1994.

[75] J. P. Lerch, J. C. Pruessner, A. Zijdenbos, H. Hampel, S. J. Teipel, and A. C. Evans, "Focal decline of cortical thickness in Alzheimer's disease identified by computational neuroanatomy," Cerebral Cortex, vol. 15, no. 7, pp. 995-1001, 2005.

[76] D. Tosun, P. Mojabi, M. W. Weiner, and N. Schuff, "Joint analysis of structural and perfusion MRI for cognitive assessment and classification of Alzheimer's disease and normal aging," NeuroImage, vol. 52, no. 1, pp. 186-197, 2010.

[77] D. H. Salat, D. N. Greve, J. L. Pacheco et al., "Regional white matter volume differences in nondemented aging and Alzheimer's disease," NeuroImage, vol. 44, no. 4, pp. 1247-1258, 2009.

[78] J. S. Damoiseaux, S. M. Smith, M. P. Witter et al., "White matter tract integrity in aging and alzheimer's disease," Human Brain Mapping, vol. 30, no. 4, pp. 1051-1059, 2009.

[79] A. Qiu, K. Oishi, M. I. Miller, C. G. Lyketsos, S. Mori, and M. Albert, "Surface-based analysis on shape and fractional anisotropy of white matter tracts in Alzheimer's disease," PloS one, vol. 5, no. 3, article e9811, 2010. 
[80] R. N. Spreng, R. A. Mar, and A. S. N. Kim, “The common neural basis of autobiographical memory, prospection, navigation, theory of mind, and the default mode: a quantitative meta-analysis," Journal of Cognitive Neuroscience, vol. 21, no. 3, pp. 489-510, 2009.

[81] R. L. Buckner, J. R. Andrews-Hanna, and D. L. Schacter, "The brain's default network: anatomy, function, and relevance to disease," Annals of the New York Academy of Sciences, vol. 1124, pp. 1-38, 2008.

[82] M. D. Greicius, G. Srivastava, A. L. Reiss, and V. Menon, “Default-mode network activity distinguishes Alzheimer's disease from healthy aging: evidence from functional MRI," Proceedings of the National Academy of Sciences of the United States of America, vol. 101, no. 13, pp. 4637-4642, 2004.

[83] D. Royall, J. H. Gao, X. Zhao, M. J. Polk, and D. Kellogg, "Asymmetric insular function predicts positional blood pressure in nondemented elderly," Journal of Neuropsychiatry and Clinical Neurosciences, vol. 21, no. 2, pp. 173-180, 2009.

[84] D. R. Royall, J. H. Gao, and D. L. Kellogg, "Insular Alzheimer's disease pathology as a cause of "age-related" autonomic dysfunction and mortality in the non-demented elderly," Medical Hypotheses, vol. 67, no. 4, pp. 747-758, 2006.

[85] L. L. Chao, N. Schuff, J. H. Kramer et al., "Reduced medial temporal lobe $\mathrm{N}$-acetylaspartate in cognitively impaired but nondemented patients," Neurology, vol. 64, no. 2, pp. 282-289, 2005.

[86] A. Pfefferbaum, E. Adalsteinsson, D. Spielman, E. V. Sullivan, and K. O. Lim, "In vivo brain concentrations of N-acetyl compounds, creatine, and choline in Alzheimer disease," Archives of General Psychiatry, vol. 56, no. 2, pp. 185-192, 1999.

[87] K. Nagata, Y. Kondoh, R. Atchison et al., "Vascular and metabolic reserve in Alzheimer's disease," Neurobiology of Aging, vol. 21, no. 2, pp. 301-307, 2000.

[88] E. M. Reiman, R. J. Caselli, K. Chen, G. E. Alexander, D. Bandy, and J. Frost, "Declining brain activity in cognitively normal apolipoprotein E $\varepsilon 4$ heterozygotes: a foundation for using positron emission tomography to efficiently test treatments to prevent alzheimer's disease," Proceedings of the National Academy of Sciences of the United States of America, vol. 98, no. 6, pp. 3334-3339, 2001.

[89] H. An and W. Lin, "Quantitative measurements of cerebral blood oxygen saturation using magnetic resonance imaging," Journal of Cerebral Blood Flow and Metabolism, vol. 20, no. 8, pp. 1225-1236, 2000.

[90] H. Lu and Y. Ge, "Quantitative evaluation of oxygenation in venous vessels using T2-relaxation-under-spin-tagging MRI," Magnetic Resonance in Medicine, vol. 60, no. 2, pp. 357-363, 2008.

[91] D. A. Yablonskiy, "Quantitation of intrinsic magnetic susceptibility-related effects in a tissue matrix. Phantom study," Magnetic Resonance in Medicine, vol. 39, no. 3, pp. 417-428, 1998.

[92] X. He and D. A. Yablonskiy, "Quantitative BOLD: mapping of human cerebral deoxygenated blood volume and oxygen extraction fraction: default state," Magnetic Resonance in Medicine, vol. 57, no. 1, pp. 115-126, 2007.

[93] X. He, M. Zhu, and D. A. Yablonskiy, "Validation of oxygen extraction fraction measurement by qBOLD technique," Magnetic Resonance in Medicine, vol. 60, no. 4, pp. 882-888, 2008. 


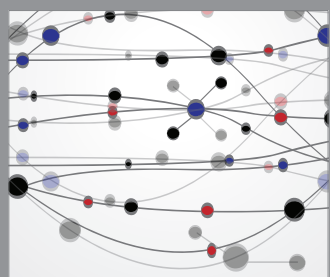

The Scientific World Journal
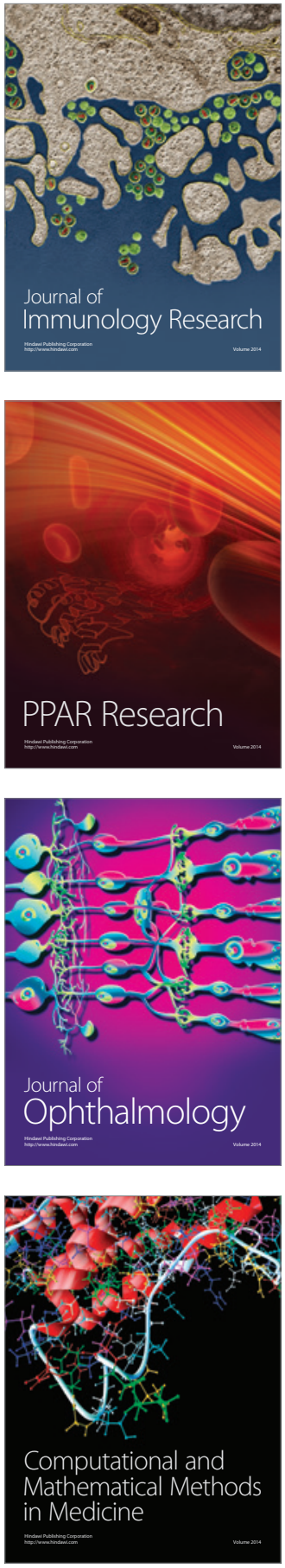

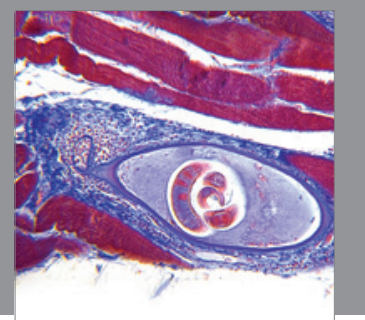

Gastroenterology

Research and Practice
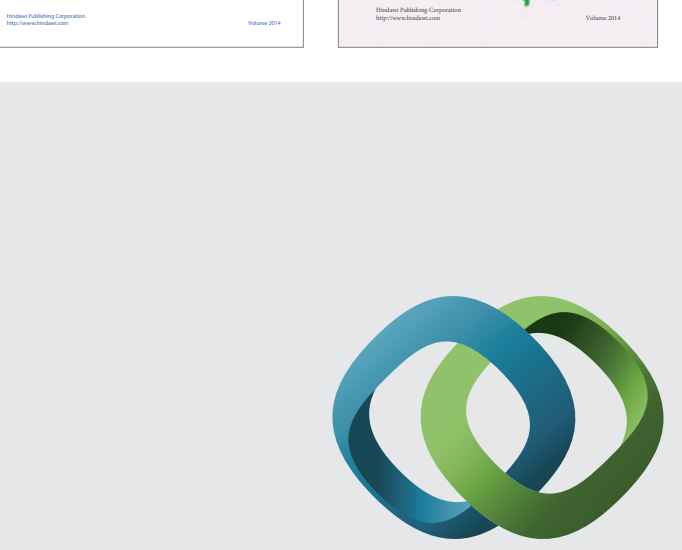

\section{Hindawi}

Submit your manuscripts at

http://www.hindawi.com
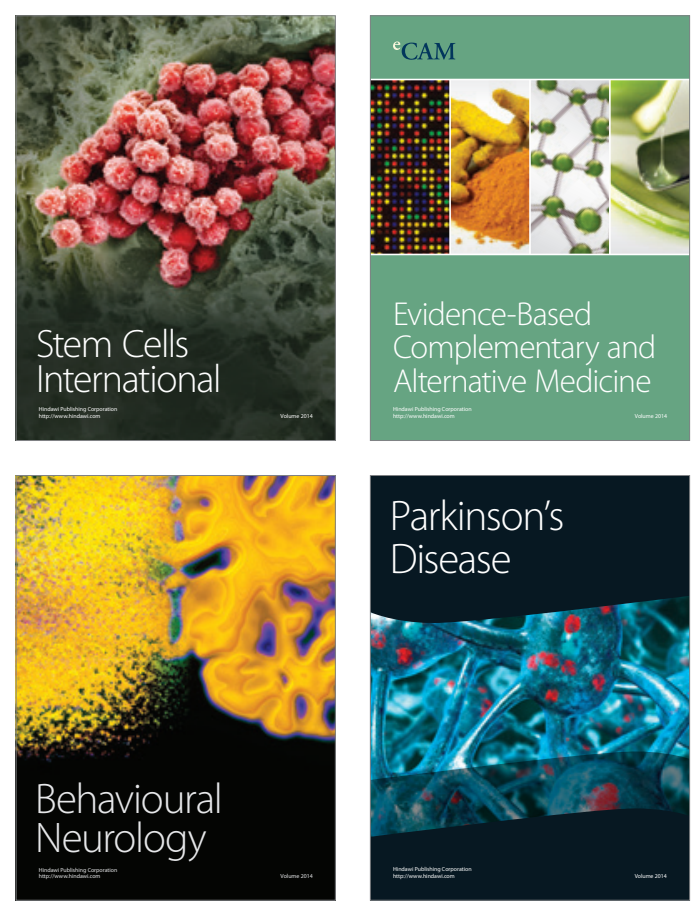

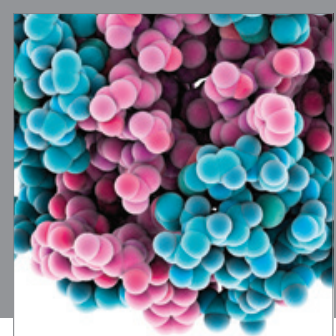

Journal of
Diabetes Research

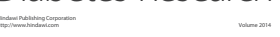

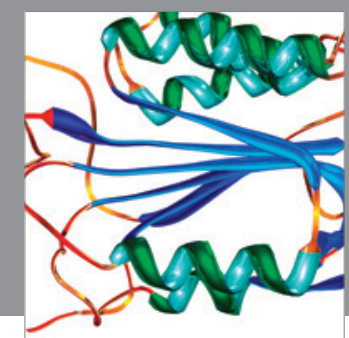

Disease Markers
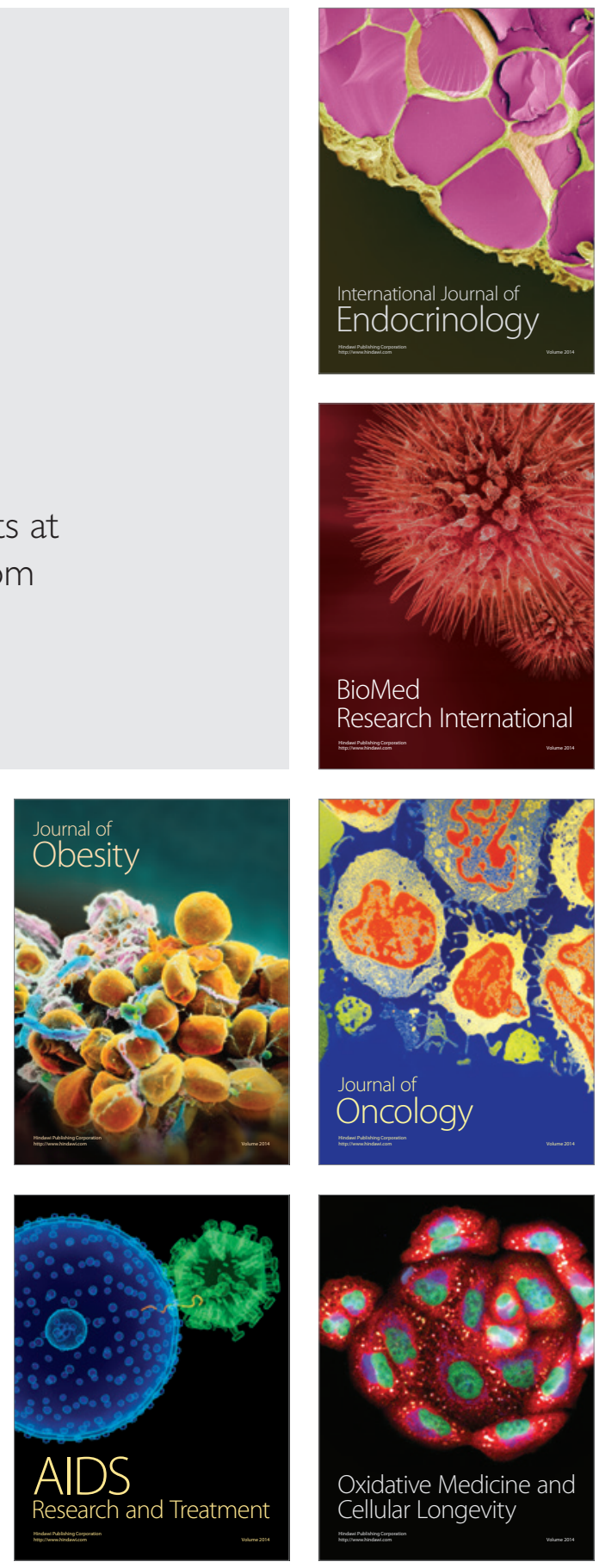